\title{
Rigidity of spherical codes
}

\author{
HENRY COHN \\ YANG JIAO \\ ABHINAV KUMAR \\ SALVATORE TORQUATO
}

\begin{abstract}
A packing of spherical caps on the surface of a sphere (that is, a spherical code) is called rigid or jammed if it is isolated within the space of packings. In other words, aside from applying a global isometry, the packing cannot be deformed. In this paper, we systematically study the rigidity of spherical codes, particularly kissing configurations. One surprise is that the kissing configuration of the Coxeter-Todd lattice is not jammed, despite being locally jammed (each individual cap is held in place if its neighbors are fixed); in this respect, the Coxeter-Todd lattice is analogous to the face-centered cubic lattice in three dimensions. By contrast, we find that many other packings have jammed kissing configurations, including the Barnes-Wall lattice and all of the best kissing configurations known in four through twelve dimensions. Jamming seems to become much less common for large kissing configurations in higher dimensions, and in particular it fails for the best kissing configurations known in 25 through 31 dimensions. Motivated by this phenomenon, we find new kissing configurations in these dimensions, which improve on the records set in 1982 by the laminated lattices.
\end{abstract}

$52 \mathrm{C} 25 ; 52 \mathrm{C} 17$

\section{Introduction}

One of the key qualitative properties of a packing is whether it is jammed, that is, whether the particles are locked into place. Jamming is of obvious scientific importance if we are using the packing to model a granular material. Furthermore, it plays a central role in studying local optimality of packings, because one natural way to try to improve a packing is to deform it so as to open up more space.

Jamming has been extensively studied for packings in Euclidean space. See, for example, Torquato and Stillinger [44] and the references cited therein. However, it has been less thoroughly investigated in other geometries. In this paper, we investigate jamming for sphere packings in spherical geometry, that is, packings of caps on the 
surface of a sphere. Jamming has previously been studied for sphere packings in $S^{2}$ (see Tarnai and Gáspár [41]), but there seems to have been little investigation in higher dimensions.

A packing of congruent spherical caps on the unit sphere $S^{n-1}$ in $\mathbb{R}^{n}$ yields a spherical code (that is, a finite subset of $S^{n-1}$ ) consisting of the centers of the caps. The minimal distance of such a code is the smallest angular separation between distinct points in the code. In other words, the cosine of the minimal distance is the greatest inner product between distinct points in the code. The packing radius is half the minimal distance, because spherical caps of this radius centered at the points of the code will not overlap, except tangentially. A spherical code is optimal if its minimal distance is as large as possible, given the dimension of the code and the number of points it contains. (Note that this notion of optimality is different from requiring that no more caps of the same size can be added without causing overlap. Neither of these two notions implies the other.)

Spherical codes arise naturally in many parts of mathematics and science (see Cohn [9] for a more extensive discussion). For example, in $\mathbb{R}^{3}$ they model pores in pollen grains or colloidal particles adsorbing to the surface of a droplet in a emulsion formed by two immiscible liquids. In higher dimensions, they can be used as error-correcting codes for a constant-power radio transmitter. Furthermore, many beautiful spherical codes arise in Lie theory, discrete geometry, or the study of the sporadic finite simple groups.

A deformation of a spherical code is a continuous motion of the points such that the minimal distance never drops below its initial value. A deformation is an unjamming if it does not simply consist of applying global isometries (that is, the pairwise distances do not all remain constant). A spherical code is called rigid or jammed if it has no unjamming. It is called locally jammed if no single point can be continuously moved while all the others are held fixed.

For example, in the face-centered cubic packing of balls in $\mathbb{R}^{3}$, the kissing configuration (that is, points of tangency on a given ball) consists of the vertices of a cuboctahedron. This code is locally jammed, but it is not in fact jammed (see Conway and Sloane [19, page 29] or Proposition 3.5 below). However, it can be deformed into an optimal spherical code, namely the vertices of a regular icosahedron, and the icosahedron is then a rigid code with a higher minimal distance than that of the cuboctahedron.

As this example shows, deforming a spherical code is one way to improve it. Some optimal codes are not jammed; for example, the best five-point codes in $S^{2}$ consist of two antipodal points and three points orthogonal to them, and the three points can move freely as long as they remain separated by at least an angle of $\pi / 2$. Furthermore, computer experiments suggest that an optimal code can have rattlers, that is, points not 
in contact with any other point, although no such case has ever been rigorously analyzed. However, despite these issues, rigidity is a powerful criterion for understanding when a code can be improved.

Note that whether a configuration is jammed depends on the ambient space. For example, the vertices of a square are jammed in $S^{1}$ but not in $S^{2}$.

For infinite packings in Euclidean space, there are more subtle distinctions between different types of jamming (see Bezdek, Bezdek and Connelly [7] and Torquato and Stillinger [43]) based on what sorts of motions are allowed. For example, are all but finitely many particles held fixed? Are shearing motions allowed? However, these issues do not arise for packings in compact spaces.

Nevertheless, jamming seems to be a more subtle phenomenon on spheres than it is in Euclidean space. In Euclidean space, there is an efficient algorithm to test for jamming (see Donev, Torquato, Stillinger and Connelly [21]) but on spheres we do not know such an algorithm. The difficulty is caused by curvature, which complicates certain arguments. For example, in Euclidean space every infinitesimal unjamming extends to an actual unjamming, as we will explain in Section 2, but the corresponding procedure does not work on spheres.

Once we have developed the basic theory of rigidity for spherical codes, we will devote the rest of this paper to applying it to analyze specific codes. We will focus primarily on kissing configurations (that is, spherical codes with minimal angle at least $\pi / 3$, or equivalently the points of tangency in Euclidean space packings), because they form a rich class of spherical codes and include many of the most noteworthy examples. An optimal kissing configuration is one with the largest possible size in its dimension.

As mentioned above, the face-centered cubic kissing configuration is not rigid, but we will prove that all of the other best configurations known in up to twelve dimensions are rigid. Along the way, we will produce what may be the first exhaustive enumeration of these configurations in up to eight dimensions, as well as a complete list of the known examples in nine through twelve dimensions (although we suspect that more remain to be discovered). Above twelve dimensions, the calculations become increasingly difficult to do, even by computer, but we analyze certain cases that are susceptible to conceptual arguments. In particular, we show that the kissing configuration of the Coxeter-Todd lattice $K_{12}$ is not rigid, while that of the Barnes-Wall lattice $\Lambda_{16}$ is, although both lattices are conjectured to be optimal sphere packings in their dimensions.

We particularly focus our attention on 25 through 31 dimensions, because of the remarkably small increases in the record kissing numbers from each dimension to the next (see Table 3 in Section 7 for the old records). The best configurations previously 


\begin{tabular}{cc|cc} 
Dimension & Kissing number & Dimension & Kissing number \\
\hline 1 & $\mathbf{2}$ & 17 & 5346 \\
2 & $\mathbf{6}$ & 18 & 7398 \\
3 & $\mathbf{1 2}$ & 19 & 10668 \\
4 & $\mathbf{2 4}$ & 20 & 17400 \\
5 & 40 & 21 & 27720 \\
6 & 72 & 22 & 49896 \\
7 & 126 & 23 & 93150 \\
8 & $\mathbf{2 4 0}$ & 24 & $\mathbf{1 9 6 5 6 0}$ \\
9 & 306 & 25 & 197040 \\
10 & 500 & 26 & 198480 \\
11 & 582 & 27 & 199912 \\
12 & 840 & 28 & 204188 \\
13 & 1154 & 29 & 207930 \\
14 & 1606 & 30 & 219008 \\
15 & 2564 & 31 & 230872 \\
16 & 4320 & 32 & 276032
\end{tabular}

Table 1: The best lower bounds known for kissing numbers in up to thirty-two dimensions. Numbers in bold are known to be optimal (see Schütte and van der Waerden [39], Levenšteľn [29], Odlyzko and Sloane [36] and Musin [34]).

known were not even locally jammed, but we see no simple way to deform them so as to increase the kissing number. However, in Section 7 we show how to improve on the known records. We give a simple argument that shows how to beat them, as well as a more complicated construction that makes use of a computer search to optimize the resulting bounds.

The new records are shown in Table 1. It is taken from Conway and Sloane [19, page xxi, Table I.2(a)], with three exceptions: the entry for $\mathbb{R}^{15}$ was out of date in that table (see [19, Chapter 5, Section 4.3]), the entries for $\mathbb{R}^{13}$ and $\mathbb{R}^{14}$ come from Zinoviev and Ericson [46], and the entries for $\mathbb{R}^{25}$ through $\mathbb{R}^{31}$ are new results in the present paper. See also Nebe and Sloane [35]. For the best upper bounds known in up to twenty-four dimensions, see Mittelmann and Vallentin [32].

Figure 1 shows a logarithmic plot of the data from Table 1, normalized for comparison with 32 dimensions. One can see the local maxima corresponding to the remarkable $E_{8}$, Barnes-Wall and Leech lattices in dimensions 8, 16 and 24, respectively. Note also that the growth rate of the known kissing numbers drops dramatically after 24 dimensions. 


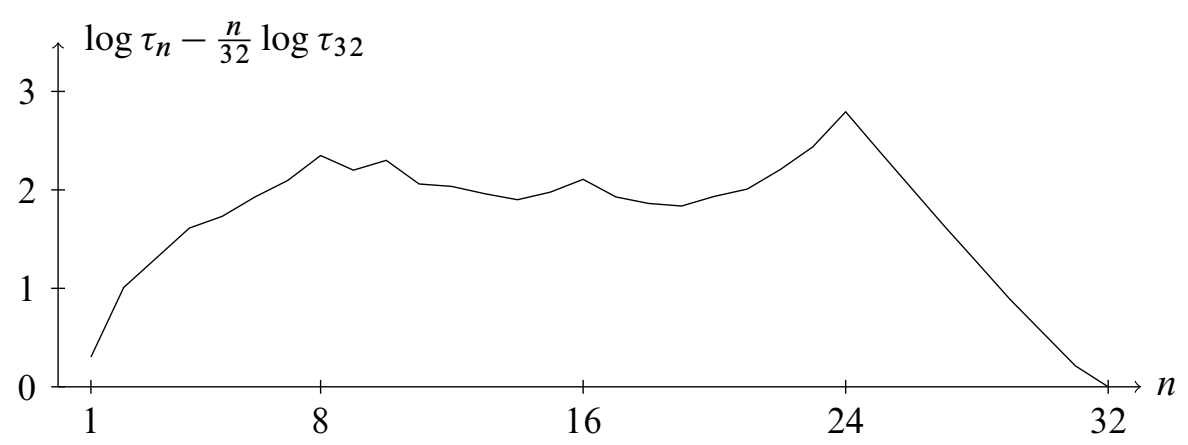

Figure 1: A plot of $\log \tau_{n}-\frac{n}{32} \log \tau_{32}$, where $\tau_{n}$ denotes the current record kissing number in $\mathbb{R}^{n}$

\section{Infinitesimal jamming}

We know of no efficient way to test whether a given spherical code is jammed. In principle, it can be done by a finite calculation, at least if the points in the code have algebraic numbers as coordinates, by using quantifier elimination for the first-order theory of the real numbers (see Tarski [42]). (The proof relies on Roth and Whiteley [38, Proposition 3.2].) However, quantifier elimination is not practical in this case.

On the other hand, there are much more efficient tests for a related concept called infinitesimal jamming, using linear programming (see Donev, Torquato, Stillinger and Connelly [21]). Given a code $\left\{x_{1}, \ldots, x_{N}\right\} \subset S^{n-1}$, imagine perturbing $x_{i}$ to $x_{i}+\varepsilon y_{i}$. Then

$$
\left|x_{i}+\varepsilon y_{i}\right|^{2}=1+2\left\langle x_{i}, y_{i}\right\rangle \varepsilon+O\left(\varepsilon^{2}\right),
$$

where $\langle\cdot, \cdot\rangle$ denotes the inner product, and

$$
\left\langle x_{i}+\varepsilon y_{i}, x_{j}+\varepsilon y_{j}\right\rangle=\left\langle x_{i}, x_{j}\right\rangle+\left(\left\langle x_{i}, y_{j}\right\rangle+\left\langle x_{j}, y_{i}\right\rangle\right) \varepsilon+O\left(\varepsilon^{2}\right) .
$$

Thus, to preserve all the constraints up to first order in $\varepsilon$, we must have $\left\langle x_{i}, y_{i}\right\rangle=0$ for all $i$, and $\left\langle x_{i}, y_{j}\right\rangle+\left\langle x_{j}, y_{i}\right\rangle \leq 0$ whenever $\left\langle x_{i}, x_{j}\right\rangle$ equals the maximal inner product in the code. An infinitesimal deformation of the code $\left\{x_{1}, \ldots, x_{N}\right\}$ is a collection of vectors $y_{1}, \ldots, y_{N}$ satisfying these constraints. It is an infinitesimal rotation if there exists a skew-symmetric matrix $\Phi \in \mathbb{R}^{n \times n}$ such that $y_{i}=\Phi x_{i}$ for all $i$, and a code is infinitesimally jammed if every infinitesimal deformation is an infinitesimal rotation. (Recall that the skew-symmetric matrices are exactly those in the Lie algebra of $S O(n)$.) Note that for an infinitesimal rotation,

$$
\left\langle x_{i}, y_{j}\right\rangle=\left\langle x_{i}, \Phi x_{j}\right\rangle=-\left\langle\Phi x_{i}, x_{j}\right\rangle=-\left\langle x_{j}, y_{i}\right\rangle \text {. }
$$


Thus, an infinitesimal rotation does not change any distances, up to first order. The converse is false (consider a square on the equator in $S^{2}$, with an infinitesimal deformation moving two opposite corners up and the other two down), but it is true for full-dimensional codes:

Lemma 2.1 Let $y_{1}, \ldots, y_{N}$ be an infinitesimal deformation of a code $\left\{x_{1}, \ldots, x_{N}\right\}$ in $S^{n-1}$ such that $x_{1}, \ldots, x_{N}$ span $\mathbb{R}^{n}$. If $\left\langle x_{i}, y_{j}\right\rangle+\left\langle x_{j}, y_{i}\right\rangle=0$ for all $i$ and $j$, then the deformation is an infinitesimal rotation.

Proof First, note that if a linear combination $\sum_{i} \alpha_{i} x_{i}$ vanishes, then $\sum_{i} \alpha_{i} y_{i}=0$ as well, because

$$
\begin{aligned}
\left\langle\sum_{i} \alpha_{i} y_{i}, x_{j}\right\rangle & =\sum_{i} \alpha_{i}\left\langle y_{i}, x_{j}\right\rangle \\
& =-\sum_{i} \alpha_{i}\left\langle x_{i}, y_{j}\right\rangle \\
& =-\left\langle\sum_{i} \alpha_{i} x_{i}, y_{j}\right\rangle \\
& =-\left\langle 0, y_{j}\right\rangle=0
\end{aligned}
$$

for all $j$ (and only the zero vector is orthogonal to a set that spans $\mathbb{R}^{n}$ ). Thus, there is a well-defined linear map $\Phi$ such that $\Phi x_{i}=y_{i}$. Furthermore, the identity

$$
\left\langle x_{i}, \Phi x_{j}\right\rangle=\left\langle x_{i}, y_{j}\right\rangle=-\left\langle y_{i}, x_{j}\right\rangle=-\left\langle\Phi x_{i}, x_{j}\right\rangle
$$

implies that $\Phi$ is skew-symmetric, because it holds for a basis of $\mathbb{R}^{n}$ and hence $\langle u, \Phi v\rangle=-\langle\Phi u, v\rangle$ for all $u, v \in \mathbb{R}^{n}$.

Every infinitesimally jammed code is in fact jammed. This is not obvious: one cannot simply differentiate a purported unjamming motion to get an infinitesimal unjamming, without dealing with two technicalities, namely whether there is a differentiable unjamming and what happens if all the first-order derivatives vanish. However, it is true, as pointed out by Connelly [13, Remark 4.1] and by Roth and Whiteley [38, Theorem 5.7]:

Theorem 2.2 (Connelly, Roth and Whiteley) Every infinitesimally jammed spherical code is jammed.

The cited papers deal with the more general setting of tensegrity frameworks, in which movable points can be connected by bars (with fixed lengths), cables (with specified maximum lengths) or struts (with specified minimum lengths), and they prove that 
infinitesimal jamming implies jamming in this setting. For the special case of spherical codes, we connect each point in the code to the origin using a bar, and we insert struts between neighboring points (that is, those at the minimal distance).

We do not know whether every jammed spherical code that spans the ambient space is infinitesimally jammed. For tensegrity frameworks, the corresponding statement is not true: if we place bars along the edges of a regular octahedron, and use two additional bars to connect its center with a pair of opposite vertices, then the framework is rigid, but flexing the center orthogonally to the two adjacent bars is a nontrivial infinitesimal deformation. We have not found such an example for spherical codes, but we expect that there is one. By contrast, infinitesimal jamming is equivalent to jamming for periodic packings in Euclidean space (see Donev, Torquato, Stillinger and Connelly [21]). Specifically, if we perturb $x$ to $x+\varepsilon y$ and $x^{\prime}$ to $x^{\prime}+\varepsilon y^{\prime}$, then

$$
\left|(x+\varepsilon y)-\left(x^{\prime}+\varepsilon y^{\prime}\right)\right|^{2}=\left|x-x^{\prime}\right|^{2}+2\left\langle x-x^{\prime}, y-y^{\prime}\right\rangle \varepsilon+\left|y-y^{\prime}\right|^{2} \varepsilon^{2} .
$$

The second-order term is always nonnegative, so nonnegativity of the first-order term suffices to produce an actual unjamming. (Deforming the underlying lattice complicates the analysis, but the result remains true; see [21, Appendix C].) What goes wrong in the spherical case is that $x+\varepsilon y$ is no longer a unit vector and must be normalized, which causes the distances to decrease.

This is not merely a technicality: there seems to be no simple method to turn an infinitesimal unjamming into an actual unjamming. Nevertheless, in all our examples, we have been able to accomplish this (with some effort).

The linear programming algorithm for infinitesimal rigidity testing works as follows. By Lemma 2.1, to test whether a full-dimensional code is infinitesimally jammed, we need only check for each pair of points whether the distance between them can be changed. In other words, in an infinitesimal deformation, are the maximum and minimum of $\left\langle x_{i}, y_{j}\right\rangle+\left\langle x_{j}, y_{i}\right\rangle$ zero for all $i$ and $j$ ? For each $i$ and $j$, this gives rise to two linear programming problems, because we are imposing linear constraints on the perturbation vectors $y_{1}, \ldots, y_{N}$ and maximizing or minimizing the linear function $\left\langle x_{i}, y_{j}\right\rangle+\left\langle x_{j}, y_{i}\right\rangle$. (Of course, when $\left\langle x_{i}, x_{j}\right\rangle$ is maximal in the code, the definition of an infinitesimal deformation requires that $\left\langle x_{i}, y_{j}\right\rangle+\left\langle x_{j}, y_{i}\right\rangle \leq 0$, so maximizing this linear functional is trivial. However, the other cases are nontrivial.) The code is infinitesimally jammed if and only if the optima in all these linear programs are zero. If not, then solving the linear programs will produce an infinitesimal unjamming, provided that we also bound the coordinates of the perturbation vectors (to avoid unbounded linear programs).

In some cases we are aided by symmetry, because we only need to check one representative from each orbit of the action of the code's symmetry group on pairs of points in the 
code. For example, if the symmetry group acts distance-transitively, then we only need to check one pair of points at each distance. Using the approach of Donev, Torquato, Stillinger and Connelly [21], we can even reduce to solving one linear program, at the cost of randomization. Specifically, consider maximizing the linear combination

$$
\sum_{i, j} c_{i, j}\left(\left\langle x_{i}, y_{j}\right\rangle+\left\langle x_{j}, y_{i}\right\rangle\right)
$$

where the coefficients $c_{i, j}$ are chosen randomly from the interval $[-1,1]$. With probability 1 , this approach will produce an infinitesimal unjamming if one exists. Thus, if the optimum is zero, then we can be confident that the code is jammed, although this does not constitute a proof.

For most of the examples in this paper, we give short conceptual proofs of jamming. However, for some cases we must rely on computer calculations. In these cases, we have given rigorous, computer-assisted proofs by using exact rational arithmetic via the QSopt_ex linear programming software of Applegate, Cook, Dash and Espinoza [1] and checking every pair of points in the code.

\section{The kissing configurations of root lattices}

We begin by proving that the root systems $D_{n}$ (for $n \geq 4$ ) and $E_{6}, E_{7}$ and $E_{8}$ are infinitesimally jammed, while $A_{n}$ is not jammed (for $n \geq 3$ ). Note that these root systems are the kissing configurations of the corresponding root lattices.

In this section, we will use spheres of diameter $\sqrt{2}$ instead of 1 , because that is standard for these root systems and makes the inner products integral. Note that the theory of infinitesimal jamming in no way depends on this normalization.

The following elementary lemma will play a key role in the proofs:

Lemma 3.1 Let $\mathcal{C}$ and $\mathcal{D}$ be spherical codes with $\mathcal{C} \subseteq \mathcal{D}$ and with the same minimal distance. If $\mathcal{C}$ is infinitesimally jammed within the vector space it spans, then in any infinitesimal deformation of $\mathcal{D}$, the inner products between points in $\mathcal{C}$ are unchanged (up to first order).

The interesting case is when $\mathcal{C}$ is lower dimensional than $\mathcal{D}$.

Proof Let $x$ and $y$ be points in $\mathcal{C}$, and let $u$ and $v$ be their perturbations in an infinitesimal deformation of $\mathcal{D}$. We write $u=u_{\mathcal{C}}+u_{\perp}$ and $v=v_{\mathcal{C}}+v_{\perp}$, where $u_{\mathcal{C}}$ and $v_{\mathcal{C}}$ are in the span of $\mathcal{C}$ while $u_{\perp}$ and $v_{\perp}$ are in the orthogonal complement of the span. 
The orthogonal projections to the span of $\mathcal{C}$ yield an infinitesimal deformation of $\mathcal{C}$. (Here we need $\mathcal{C}$ and $\mathcal{D}$ to have the same minimal distance, since otherwise the conditions on which inner products can increase will differ.) Thus, because $\mathcal{C}$ is infinitesimally jammed within its span, $\left\langle x, v_{\mathcal{C}}\right\rangle+\left\langle u_{\mathcal{C}}, y\right\rangle=0$. Furthermore, $\left\langle x, v_{\perp}\right\rangle=$ $\left\langle y, u_{\perp}\right\rangle=0$. It follows that $\langle x, v\rangle+\langle u, y\rangle=0$, as desired.

Lemma 3.2 The $A_{2}$ root system is infinitesimally jammed.

The $A_{2}$ root system is a regular hexagon, and it is easy to show that every regular polygon is infinitesimally jammed. This simple observation provides a useful tool for analyzing more elaborate configurations via Lemma 3.1.

Proposition 3.3 The $D_{4}$ root system is infinitesimally jammed.

Proof The minimal vectors of $D_{4}$ have norm 2 and the possible inner products between distinct minimal vectors are $0, \pm 1$ and -2 . First, note that the automorphism group of $D_{4}$ acts transitively on pairs of minimal vectors with a given inner product, so without loss of generality we can consider just one pair of points at each distance. (This transitivity fails for $D_{n}$ with $n>4$, because there are two orbits for inner product 0 , but the triality symmetry of $D_{4}$ collapses them to one orbit.)

Furthermore, $D_{4}$ contains $A_{2}$, and by Lemma 3.1 the distances in a copy of $A_{2}$ cannot change because $A_{2}$ is infinitesimally jammed within its span. This takes care of all the cases except for a pair of orthogonal vectors.

We now have to show that if $\langle x, y\rangle=0$ then $\langle x, y\rangle$ does not change in any infinitesimal deformation. Again by the distance transitivity of the automorphism group, we may assume $x=(1,1,0,0)$ and $y=(1,-1,0,0)$. Let $u=(1,0,1,0), v=(1,0,-1,0)$, $w=(1,0,0,1)$ and $z=(1,0,0,-1)$ be other minimal vectors of $D_{4}$. Denote the first order perturbations of $x, y, u, v, w, z$ by $x^{\prime}, y^{\prime}, u^{\prime}, v^{\prime}, w^{\prime}, z^{\prime}$. Note that $x+y=$ $u+v=w+z$, and that $\langle x, u\rangle=\langle x, v\rangle=\langle y, u\rangle=\langle y, v\rangle=1$. Thus, by the $A_{2}$ embedding argument, we have

$$
\begin{aligned}
\left\langle x, u^{\prime}\right\rangle+\left\langle x^{\prime}, u\right\rangle & =0, & \left\langle y, u^{\prime}\right\rangle+\left\langle y^{\prime}, u\right\rangle & =0, \\
\left\langle x, v^{\prime}\right\rangle+\left\langle x^{\prime}, v\right\rangle & =0, & \left\langle y, v^{\prime}\right\rangle+\left\langle y^{\prime}, v\right\rangle & =0 .
\end{aligned}
$$

Adding these equations, we get

$$
\left\langle x+y, u^{\prime}+v^{\prime}\right\rangle+\left\langle x^{\prime}+y^{\prime}, u+v\right\rangle=0,
$$

or (using $x+y=u+v$ )

$$
\left\langle u+v, u^{\prime}+v^{\prime}\right\rangle+\left\langle x^{\prime}+y^{\prime}, x+y\right\rangle=0 .
$$


Since we know that $\left\langle u, u^{\prime}\right\rangle=0$, etc., we get (denoting the first order change $\left\langle u, v^{\prime}\right\rangle+$ $\left\langle u^{\prime}, v\right\rangle$ in $\langle u, v\rangle$ by $\left.\delta(u, v)\right)$

$$
\delta(u, v)+\delta(x, y)=0 .
$$

Similarly, we have

$$
\begin{aligned}
& \delta(u, v)+\delta(w, z)=0, \\
& \delta(w, z)+\delta(x, y)=0 .
\end{aligned}
$$

From these three equations, elementary algebra implies $\delta(x, y)=\delta(u, v)=\delta(z, w)=0$. This completes the proof.

The lengthy argument for the last case amounts to verifying that a square embedded within $D_{4}$ cannot be infinitesimally deformed. Note that this cannot simply be settled using Lemma 3.1, although the square is indeed jammed within its span, because the minimal distance in the square differs from that in $D_{4}$. If that argument worked, it would also prove infinitesimal jamming for $D_{3}$, which is not true. (The $A_{3}$ and $D_{3}$ root lattices are isomorphic to the face-centered cubic lattice, whose kissing configuration is not jammed.)

Corollary 3.4 The $D_{n}$ root system (for $n \geq 4$ ) and the $E_{6}, E_{7}$ and $E_{8}$ root systems are infinitesimally jammed.

Proof These configurations have norm 2 and inner products $0, \pm 1$ and \pm 2 , the same as in $D_{4}$. We first deal with $E_{6}, E_{7}$ and $E_{8}$. Their automorphism groups act distance transitively, so it suffices to consider a single pair of points at each distance. The $D_{4}$ root system embeds in each of these configurations (in fact, its Dynkin diagram is a subdiagram), so without loss of generality we can assume the pair of points is in $D_{4}$. Now combining Lemma 3.1 and Proposition 3.3 completes the proof.

The same proof works for $D_{n}$ with $n>4$, with one exception, namely that there are two orbits of pairs of orthogonal vectors, so the group does not quite act distance transitively. Specifically, the stabilizer of $(1,1,0, \ldots, 0)$ cannot interchange $(1,-1,0, \ldots, 0)$ and $(0,0,1,1,0, \ldots, 0)$. However, in both cases, these vectors are contained in a copy of $D_{4}$ (namely, the one in the first four coordinates), so we can complete the proof as before.

The $A_{n}$ root system is locally jammed, and for $n=2$ it is in fact jammed, but the unjamming for $n=3$ extends to higher dimensions.

Proposition 3.5 For $n \geq 3$, the $A_{n}$ root system is not jammed. 
Proof We will demonstrate an explicit unjamming. We realize the $A_{n}$ lattice as the set of integral vectors in the subspace $\left\{\left(x_{0}, \ldots, x_{n}\right): \sum_{i} x_{i}=0\right\}$ of $\mathbb{R}^{n+1}$, so each vector in the $A_{n}$ root system has one 1 and one -1 among its coordinates.

We begin with $A_{3}$, whose twelve vertices form a cuboctahedron with six square facets and eight triangular facets, and we choose a perfect matching using non-overlapping diagonals of the squares. In the left half of the diagram below, we have labeled matched vertices with the same label (from 1 to 6). Each matched pair $v$ and $v^{\prime}$ satisfies $\left\langle v, v^{\prime}\right\rangle=0$, so we can perturb $v$ to $\left(v+\varepsilon v^{\prime}\right) / \sqrt{1+\varepsilon^{2}}$ and $v^{\prime}$ to $\left(v^{\prime}+\varepsilon v\right) / \sqrt{1+\varepsilon^{2}}$, as shown on the right.
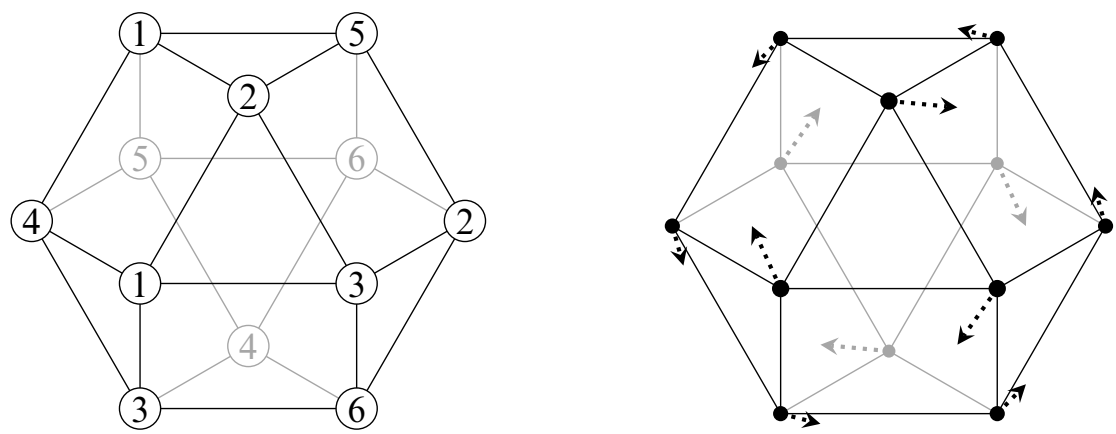

To see that this is an actual unjamming, we must check what happens to vertices $v$ and $w$ satisfying $\langle v, w\rangle=1$. The matched vertices satisfy $\left\langle v^{\prime}, w^{\prime}\right\rangle=-1$ and $\left\langle v^{\prime}, w\right\rangle=-\left\langle v, w^{\prime}\right\rangle$, and hence

$$
\frac{\left\langle v+\varepsilon v^{\prime}, w+\varepsilon w^{\prime}\right\rangle}{1+\varepsilon^{2}}=\frac{1-\varepsilon^{2}}{1+\varepsilon^{2}}<1,
$$

as desired.

For $A_{n}$ with $n>3$, we simply perform this unjamming on the copy of $A_{3}$ in the first four coordinates, while leaving all the other points unchanged. All we need to check is that the minimal distances between the moving points and the unchanged points do not decrease. Suppose $v$ is in $A_{3}$, paired with $v^{\prime}$ as above, while $w$ is outside $A_{3}$ and satisfies $\langle v, w\rangle=1$. Then $v$ and $w$ have a single nonzero coordinate in which they agree, and the other nonzero coordinate of $w$ is not among the first four coordinates. Because $\left\langle v, v^{\prime}\right\rangle=0$, the vectors $v$ and $v^{\prime}$ have disjoint supports, from which it follows that $\left\langle v^{\prime}, w\right\rangle=0$. Thus,

$$
\frac{\left\langle v+\varepsilon v^{\prime}, w\right\rangle}{\sqrt{1+\varepsilon^{2}}}=\frac{1}{\sqrt{1+\varepsilon^{2}}}<1,
$$

so we have a genuine unjamming of the $A_{n}$ root system. 
Note that the first-order changes in the inner products of 1 vanish, as they must according to Lemma 3.1 (because each pair of vectors with inner product 1 spans a hexagon); instead, there is a second-order improvement.

\section{Kissing configurations in five, six and seven dimensions}

The root systems analyzed in the previous section are conjectured to be optimal kissing configurations up through eight dimensions. In fact, $E_{8}$ is known to be the unique optimal kissing configuration in $\mathbb{R}^{8}$ (optimality was proved by Levenšteĭn [29] and by Odlyzko and Sloane [36], while uniqueness was proved by Bannai and Sloane [2]). However, in five, six and seven dimensions, the $D_{5}, E_{6}$ and $E_{7}$ root systems are not the unique optimal kissing configurations. This was first observed by Leech [27; 28].

In this section, we enumerate what we believe may be a complete list of optimal kissing configurations in these dimensions, up to isometry. We find two configurations in $\mathbb{R}^{5}$, four in $\mathbb{R}^{6}$, and four in $\mathbb{R}^{7}$. Those in $\mathbb{R}^{5}$ and $\mathbb{R}^{6}$, as well as two of the ones in $\mathbb{R}^{7}$, are all kissing configurations of previously known sphere packings. However, two of the configurations in $\mathbb{R}^{7}$ do not arise naturally from densest packings in $\mathbb{R}^{7}$ and appear to be new.

We have verified by rigorous computation calculations that all these configurations are infinitesimally jammed. (It would very likely be possible to check this by hand, but it does not seem worth the effort.) To do so, we used version 2.6 of the QSopt_ex rational LP solver [1] to get exact solutions to the linear programs. Specifically, for each pair of points, we used the software to verify that their inner product can neither increase nor decrease in any infinitesimal deformation. Of course, it would be far more efficient to take into account the orbits of the automorphism group on pairs of points, but it was easier to let the computer check many cases than to compute and keep track of the orbits. The one tricky part of the calculation is that the coordinates of the points are not always rational numbers (and using floating-point arithmetic would make the calculations unrigorous). Fortunately, there is an easy fix: by rescaling certain coordinates, in each case we can use rational coordinates and carry out all the calculations with respect to a nonstandard inner product defined by a rational matrix. In fact, we can take the matrix to be diagonal. In the supplementary information for this paper (see Appendix A), we provide explicit rational coordinates for all of the configurations studied in this section, together with the corresponding inner product matrices.

\subsection{Methods}

Conway and Sloane [18] describe a method that conjecturally generates all the best sphere packings in low dimensions. For dimensions from five to eight, it works as 
follows. We start with the checkerboard lattice

$$
D_{4}=\left\{x \in \mathbb{Z}^{4}: \sum_{i=1}^{4} x_{i} \equiv 0(\bmod 2)\right\} .
$$

It leads to a sphere packing in $\mathbb{R}^{4}$ with balls of radius $\sqrt{2} / 2$, which is conjecturally the densest sphere packing in four dimensions, as well as the unique densest periodic packing. The discriminant group $D_{4}^{*} / D_{4} \cong(\mathbb{Z} / 2 \mathbb{Z})^{2}$ has four elements, the zero class $a=(0,0,0,0)$ and three nonzero classes, which we represent by the deep holes $b=(1,0,0,0), c=(1 / 2,1 / 2,1 / 2,1 / 2)$ and $d=(-1 / 2,1 / 2,1 / 2,1 / 2)$. Conway and Sloane conjecture that the densest packings in dimension $n$ (with $5 \leq n \leq 8$ ) are obtained by fibering over $D_{4}$, that is, by positioning translated copies of $D_{4}$ over the densest packings in dimension $n-4$, scaled so that their minimal distance is 1. Each translation vector will be one of $a, b, c$ or $d$, and adjacent points in the $(n-4)$-dimensional packing will be assigned different translation vectors. This idea of fibering is a generalization of the construction of laminated lattices (see Conway and Sloane [15]). See also Cohn and Kumar [11] for other calculations using the methods of [18].

\subsection{Dimension 5: 40 points on $S^{4}$}

In dimension 5, the densest packings are conjecturally obtained by stacking layers of $D_{4}$ on top of each other, that is, by arranging translated copies of $D_{4}$ along the integers $\mathbb{Z}$. At each point of $\mathbb{Z}$, one has to make a choice of which translate of $D_{4}$ to use. In other words, such a packing corresponds to a coloring of $\mathbb{Z}$ by four colors or labels $a, b, c, d$, so that no two adjacent integers have the same color.

For instance, the $D_{5}$ lattice is obtained from the following coloring:

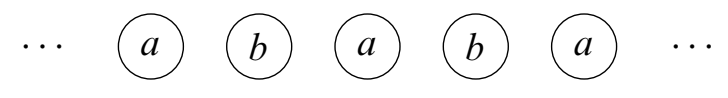

Because the affine symmetry group of $D_{4}^{*}$ acts as the full symmetric group on the four classes in $D_{4}^{*} / D_{4}$, it does not matter which two distinct classes are used as labels here.

The best kissing configurations known in dimension 5 are obtained from the local versions of these packings. The $D_{5}$ root system comes from the following diagram:

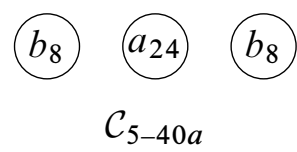


The subscripts mean that we take the 24 minimal lattice vectors in the central $D_{4}$ layer, and 8 translated lattice vectors in each adjacent layer (specifically, those surrounding a deep hole). We call this configuration $\mathcal{C}_{5-40 a}$.

Of course, there is no need to choose the same translation vector on both sides of the central layer, and we can form a competing configuration $\mathcal{C}_{5-40 b}$ as follows:

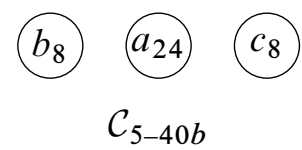

This is the kissing configuration of the other three uniform 5-dimensional packings described in [18]. It is genuinely different from $\mathcal{C}_{5-40 a}$, because it lacks antipodal symmetry. Its symmetry group has size 384 , compared to 3840 for $\mathcal{C}_{5-40 a}$.

\subsection{Dimension 6: 72 points on $S^{5}$}

Next, we consider kissing configurations in six dimensions. The best packings known in $\mathbb{R}^{6}$ are obtained by fibering over $D_{4}$ using a hexagonal arrangement of translates in the plane. There are four uniform packings, described concisely by the colors associated to the hexagon of six translates around a central copy of $D_{4}$. For more details we refer the reader to Conway and Sloane [18], and Cohn and Kumar [11].

These packings have four distinct kissing configurations, shown in Figure 2, and a simple case analysis shows that every possibility in this framework is equivalent to one of them (up to symmetries of the hexagon and permutations of the labels). They are genuinely different, as their automorphism groups have sizes 103680, 3840, 2304 and 384 , respectively.

\subsection{Dimension 7: 126 points on $S^{6}$}

The best packings known in $\mathbb{R}^{7}$ are obtained by fibering over $D_{4}$ in an optimal 3dimensional arrangement, namely one of the Barlow packings. The Barlow packings are themselves obtained by stacking translates of the hexagonal lattice. Here, we will be concerned with the kissing configurations, which involve only three hexagonal layers, so although there are uncountably many Barlow packings (corresponding to three-colorings of $\mathbb{Z}$ ), we will only need to consider two configurations: the hexagonal close-packing, in which the top and bottom layers are mirror images of each other, and the face-centered cubic packing, in which they are point reflections of each other.

First, we consider the face-centered cubic arrangement. Without loss of generality we label the central sphere at the origin $a$. Once we have specified the labels in the 


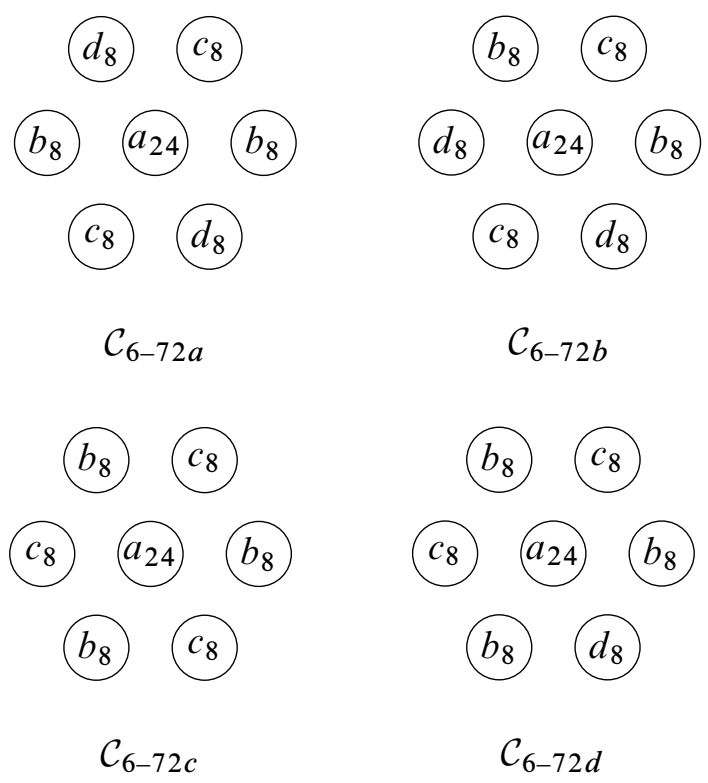

Figure 2: Kissing configurations in $\mathbb{R}^{6}$ based on the hexagonal lattice

central hexagonal layer, the labels on the minimal vectors in the adjacent layers will be completely determined (since they will each already have three neighbors with different labels). It is not hard to check that in the central layer, the hexagon surrounding the central copy of $D_{4}$ must be colored either $b c d b c d$ or $b c b d c d$, up to a permutation of the colors, because no other choices will extend consistently to the neighboring layers. This determines all the labels in the two diagrams shown in Figure 3, except for the six points labeled $a_{1}$. For those points, one can check that if their labels agree with the label assigned to the central sphere, then they add one to the kissing number; otherwise they add zero. Thus, they should all be labeled $a$ to get a kissing number of 126 .

Accordingly, we get two local configurations of tight packings, as shown in Figure 3. The code $\mathcal{C}_{7-126 a}$ is the $E_{7}$ root system, but $\mathcal{C}_{7-126 b}$ is not the kissing configuration of any of the tight packings in $\mathbb{R}^{7}$ described by Conway and Sloane [18].

The two other conjecturally optimal kissing arrangements in $S^{6}$ come from the hexagonal close-packing via a similar argument and are shown in Figure 4 . The code $\mathcal{C}_{7-126}$ is the kissing configuration of the packing $\Lambda_{7}^{2}$ described in [18], while $\mathcal{C}_{7-126 d}$ does not occur in any of the tight packings analyzed in that paper.

These four spherical codes can again be distinguished by the sizes of their automorphism groups, which are 2903040, 46080, 103680 and 3840, respectively. 


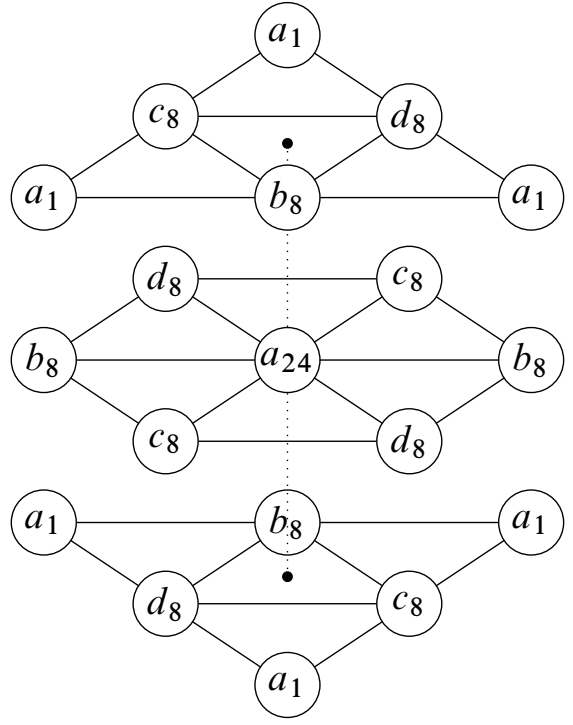

$\mathcal{C}_{7-126 a}$
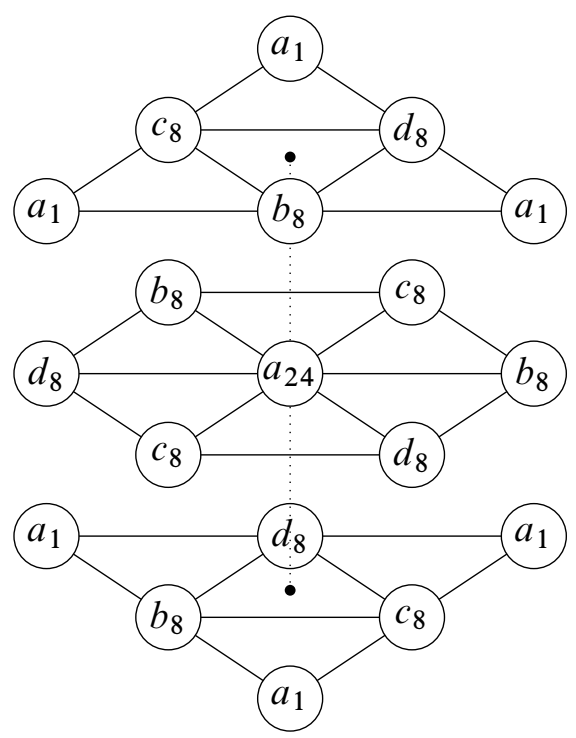

$\mathcal{C}_{7-126 b}$

Figure 3: Kissing configurations in $\mathbb{R}^{7}$ based on the face-centered cubic packing

\section{Nine through twelve dimensions}

Up through eight dimensions, we are quite confident that the known kissing configurations are optimal and reasonably confident that the list we have provided is complete. However, the situation in nine or more dimensions is very different. For example, in $\mathbb{R}^{9}$ the highest kissing number achieved by any lattice is 272 (by a theorem of Watson [45]), while the best kissing number known is 306. Furthermore, there is little reason to believe that the usual constructions based on error-correcting codes (see Conway and Sloane [19, Chapter 5]) will prove adequate in high dimensions. Even in $\mathbb{R}^{10}$, computer searches have led to intriguing new kissing configurations (see Elser and Gravel [24]), although so far they have not improved on the known records.

The laminated lattice $\Lambda_{9}$ achieves kissing number 272 , and it is the only lattice that does. Here, we prove two properties of this kissing configuration: it is not locally jammed, but it is the largest possible kissing configuration in $\mathbb{R}^{9}$ that contains the $E_{8}$ root system as a cross section.

To describe this code, we first recall the structure of the root lattice $E_{8}$, which is used to produce $\Lambda_{9}$. It the union of two translates of $D_{8}$, namely

$$
E_{8}=D_{8} \cup\left(D_{8}+(1 / 2, \ldots, 1 / 2)\right) .
$$



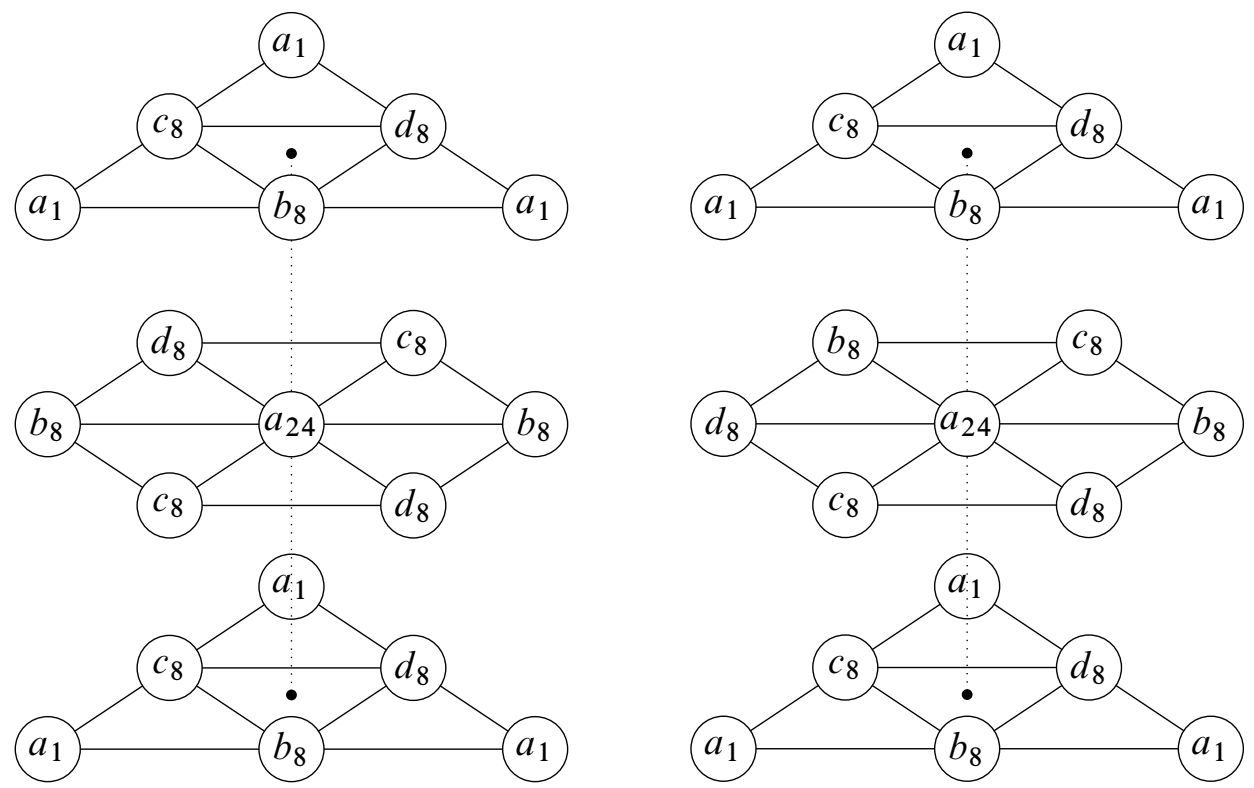

$\mathcal{C}_{7-126 c}$

$\mathcal{C}_{7-126 d}$

Figure 4: Kissing configurations in $\mathbb{R}^{7}$ based on the hexagonal close-packing

The minimal vectors of $D_{8}$ are the 112 vectors of the form $\pm e_{i} \pm e_{j}$ with $i \neq j$ (where $e_{i}$ is the $i$ th standard unit vector), and the $E_{8}$ kissing configuration consists of these vectors as well as the 128 vectors of the form $( \pm 1 / 2, \ldots, \pm 1 / 2)$ with an even number of minus signs.

The vector $v=(1,0,0, \ldots, 0)$ is a deep hole of $E_{8}$, and the lattice $\Lambda_{9}$ is generated by $E_{8} \times\{0\}$ and $v \times\{1\}$ in $\mathbb{R}^{9}$. Its kissing configuration consists of 272 vectors, and is the union of the kissing configuration $S$ of $D_{9}$ (which contains 144 vectors) and the set $T$ of 128 vectors of the form $( \pm 1 / 2, \ldots, \pm 1 / 2,0)$ with an even number of minus signs.

By Corollary 3.4, the subconfiguration $S$ is itself jammed, so it is futile to move those points of the code, but we will show that the points in $T$ are not even locally jammed. The reason is that they have no neighbors outside of $E_{8} \times\{0\}$, so they are free to move orthogonally to $E_{8}$.

More formally, given $(x, 0) \in T$ with $x \in E_{8} \backslash D_{8}$, we move it to $(x \cos \theta, \sqrt{2} \sin \theta)$, where $-\pi / 2 \leq \theta \leq \pi / 2$. For every point $y \in E_{8}$ with $y \neq x$, we have $\langle x, y\rangle \leq 1$ and hence

$$
\langle(x \cos \theta, \sqrt{2} \sin \theta),(y, 0)\rangle=\langle x, y\rangle \cos \theta \leq 1 .
$$


Therefore, the only points $(x \cos \theta, \sqrt{2} \sin \theta)$ can come too close to are those in $D_{9} \backslash D_{8}$, that is, those of the form $\pm e_{i} \pm e_{9}$ with $1 \leq i \leq 8$. We do indeed run into problems from these points when $|\theta|$ is too large, but not when $|\theta|$ is small. The relevant inner product is

$$
\left\langle(x \cos \theta, \sqrt{2} \sin \theta), \pm e_{i} \pm e_{9}\right\rangle= \pm \frac{1}{2} \cos \theta \pm \sqrt{2} \sin \theta .
$$

When $|\theta|$ is sufficiently small, the right side is clearly bounded by 1 . Specifically, we obtain a bound of 1 whenever

$$
|\theta| \leq \alpha \stackrel{\text { def }}{=} \tan ^{-1} \frac{\sqrt{5}-\sqrt{2}}{2} \approx 0.124 \pi
$$

Thus, the point $(x, 0)$ is not locally jammed.

Furthermore, we can even simultaneously move all the points of $T$ by moving $(x, 0)$ to $\left(x \cos \theta_{x}, \sqrt{2} \sin \theta_{x}\right)$, where $-\alpha \leq \theta_{x} \leq \alpha$ and $\theta_{x}$ has sign $(-1)^{k_{x} / 2}$, with $k_{x}$ being the number of negative coordinates of $x$. All we need to check is whether these points come too close to each other. The inner product between two of them is simply $\left\langle\left(x \cos \theta_{x}, \sqrt{2} \sin \theta_{x}\right),\left(y \cos \theta_{y}, \sqrt{2} \sin \theta_{y}\right)\right\rangle=\langle x, y\rangle\left(\cos \theta_{x}\right)\left(\cos \theta_{y}\right)+2\left(\sin \theta_{x}\right)\left(\sin \theta_{y}\right)$. If $\langle x, y\rangle=1$, then $x$ and $y$ have differing signs in two coordinates (that is, $k_{x}$ and $k_{y}$ differ by 2 ). In that case $\theta_{x}$ and $\theta_{y}$ have opposite signs, so

$$
\left(\cos \theta_{x}\right)\left(\cos \theta_{y}\right)+2\left(\sin \theta_{x}\right)\left(\sin \theta_{y}\right) \leq\left(\cos \theta_{x}\right)\left(\cos \theta_{y}\right) \leq 1 .
$$

On the other hand, if $\langle x, y\rangle \leq 0$, then the inner product is at most $2\left(\sin \theta_{x}\right)\left(\sin \theta_{y}\right)$, which is again at most 1 (because $\left|\theta_{x}\right|$ and $\left|\theta_{y}\right|$ are less than $\pi / 4$ ).

Proposition 5.1 No kissing configuration in $\mathbb{R}^{9}$ that contains the $E_{8}$ root system as a cross section can have more than 272 points.

Proof Suppose $\mathcal{C}$ is a kissing configuration that contains the points $(x, 0)$ for $x \in E_{8}$ with $|x|^{2}=2$. The remaining points must be of the form $(y \cos \theta, \sqrt{2} \sin \theta)$ with $y \in \mathbb{R}^{8}$ satisfying $|y|^{2}=2$ and $-\pi / 2 \leq \theta \leq \pi / 2$.

Given any such point $y$, there exists a minimal vector $x \in E_{8}$ such that $\langle x, y\rangle \geq \sqrt{2}$. This claim amounts to knowing the depth of the deep holes in the $E_{8}$ root system, which correspond to the cross-polytope facets of its convex hull. (See, for example, Conway and Sloane [17].) It follows that $\theta=0$ is impossible, since $(y, 0)$ would come too close to the point $(x, 0)$. In fact, because the points $(x, 0)$ and $(y \cos \theta, \sqrt{2} \sin \theta)$ in $\mathcal{C}$ must have inner product at most 1 , we must have $\sqrt{2} \cos \theta \leq 1$. Thus, $|\sin \theta| \geq 1 / \sqrt{2}$. Without loss of generality, we will focus on the points with $\sin \theta \geq 1 / \sqrt{2}$. 
Given two distinct such points $(y \cos \theta, \sqrt{2} \sin \theta)$ and $(z \cos \varphi, \sqrt{2} \sin \varphi)$, their inner product is again at most 1 . Therefore,

$$
1 \geq\langle y, z\rangle \cos \theta \cos \varphi+2 \sin \theta \sin \varphi \geq\langle y, z\rangle \cos \theta \cos \varphi+1,
$$

from which it follows that $\langle y, z\rangle \leq 0$. (Note that $\cos \theta$ and $\cos \varphi$ cannot vanish, since then the sine term would be too large.)

There are no more than 16 vectors in $S^{7}$ for which all inner products between distinct vectors are nonpositive (see Böröczky [8, Theorem 6.2.1]). Thus, we have shown that $\mathcal{C}$ contains at most 16 additional vectors in each hemisphere, for a total of at most $240+2 \cdot 16=272$, as desired.

As a consequence, the best kissing configuration in $\mathbb{R}^{9}$ cannot contain the best one in $\mathbb{R}^{8}$ (namely, $E_{8}$ ) as a cross section. Note also that in any 272-point kissing configuration containing $E_{8}$, equality holds throughout the proof of Proposition 5.1, so there are only finitely many such configurations, each consisting of $E_{8}$ with cross polytopes sitting above and below some of its deep holes. However, the relative position of these cross polytopes may vary. For example, in $\Lambda_{9}$ one of the cross polytopes consists of the points $\pm e_{i}-e_{9}$ with $1 \leq i \leq 8$. If $H$ is any $4 \times 4$ Hadamard matrix, we could replace this cross polytope with the points $\pm v_{i}-e_{9}$, where $v_{1}, \ldots, v_{8}$ are the rows of the matrix

$$
\left[\begin{array}{cc}
H / 2 & 0 \\
0 & H / 2
\end{array}\right]
$$

The resulting code is genuinely different, because it contains two points with inner product $-3 / 2$, while only $\pm 2, \pm 1, \pm 1 / 2$ and 0 occur in the $\Lambda_{9}$ configuration.

Table 2 lists the best kissing numbers known in dimensions 9 through 12 (see Conway and Sloane [19, pages 139-140]). The configurations are constructed using constant weight binary codes, and in fact the $E_{7}$ and $E_{8}$ root systems can also be constructed in this way. Every binary code $\mathcal{B}$ of block length $n$, size $N$, constant weight 4 and minimal distance 4 yields a periodic packing in $\mathbb{R}^{n}$, namely all the vectors in $\mathbb{Z}^{n}$ that reduce to codewords in $\mathcal{B}$ modulo 2 . The vectors of norm 4 in this packing form a kissing configuration of size $2 n+16 N$, consisting of the points $\pm 2 e_{i}$ together with signed codewords from $\mathcal{B}$ (that is, vectors with arbitrary \pm 1 entries in the support of a codeword).

For $n=9,10,11$ and 12 one can achieve $N=18,30,35$ and 51, respectively. These codes are unique up to isomorphism when $n=9$ or 10 , there are 11 of them for $n=11$, and there are 17 of them for $n=12$ (see [37, Table I]; the $n=9$ case was proved by Östergård [37], the $n=10$ case by Barrau [3], and the $n=11$ and 12 


\begin{tabular}{ccc} 
Dimension & Best known kissing number & Packing \\
\hline 9 & 306 & $P_{9 a}$ \\
10 & 500 & $P_{10 b}$ \\
11 & 582 & $P_{11 c}$ \\
12 & 840 & $P_{12 a}$
\end{tabular}

Table 2: The best kissing numbers known in dimensions 9 through 12

cases by Best $[4 ; 5 ; 6])$. Data files giving coordinates for these codes are available in the supplementary information (see Appendix A).

For $n \geq 11$ there are multiple codes, and each of these binary codes yields a distinct kissing configuration. To see why, first observe that each contains a cross polytope consisting of the vectors of the form $\pm 2 e_{i}$. This cross polytope is uniquely distinguished by the property of having large valencies. Specifically, when $n=11$ it consists of exactly the points with at least 88 neighbors (that is, points at inner product 2), and when $n=12$ it consists of those with at least 136 neighbors. These distinguished cross polytopes must correspond under any isomorphism between two configurations, which must therefore be a signed permutation of the coordinates. However, it would then yield an isomorphism of the underlying binary codes.

In nine through twelve dimensions, these kissing configurations are all jammed, due to the following proposition, whose hypotheses can speedily be checked by a computer calculation:

Proposition 5.2 Let $\mathcal{B}$ be a constant weight code of block length $n$, weight 4 and minimal distance 4 . If $\mathcal{B}$ has the following two properties, then the corresponding kissing configuration in $\mathbb{R}^{n}$ is infinitesimally jammed:

(1) For $i<j$, there is a codeword $x=\left(x_{1}, \ldots, x_{n}\right) \in \mathcal{B}$ such that $x_{i}=x_{j}=1$.

(2) For every $x \in \mathcal{B}$ and $i$ such that $x_{i}=0$, there exists $y \in \mathcal{B}$ such that $y_{i}=1$ and $d(x, y)=4$ (that is, the supports of $x$ and $y$ overlap in exactly two coordinates).

Proof Let $\mathcal{C}$ be the kissing configuration obtained from $\mathcal{B}$. First, observe that for $i \neq j$, the points $2 e_{i}$ and $2 e_{j}$ are part of a scaled $D_{4}$ root system embedded in $\mathcal{C}$. Specifically, by (1), there exist $k$ and $\ell$ such that $\pm e_{i} \pm e_{j} \pm e_{k} \pm e_{\ell}$ are all in $\mathcal{C}$; then

$$
\left\{ \pm 2 e_{i}, \pm 2 e_{j}, \pm 2 e_{k}, \pm 2 e_{\ell}, \pm e_{i} \pm e_{j} \pm e_{k} \pm e_{\ell}\right\}
$$

is a scaled $D_{4}$ root system. Therefore, by Lemma 3.1 and Proposition 3.3, the inner products in $D_{4}$ and thus in the cross polytope $\left\{ \pm 2 e_{i}: 1 \leq i \leq n\right\}$ cannot change in any infinitesimal deformation. Since the cross polytope spans $\mathbb{R}^{n}$, we can assume that the points in it are fixed. 
Let

$$
x=s_{i} e_{i}+s_{j} e_{j}+s_{k} e_{k}+s_{\ell} e_{\ell} \in \mathcal{C}
$$

be any of the remaining vectors, with $s_{i}, s_{j}, s_{k}, s_{\ell} \in\{ \pm 1\}$, and let $y$ be the infinitesimal perturbation of $x$. Then $x$ and $2 e_{i}$ generate a scaled copy of the $A_{2}$ root system in $\mathcal{C}$, which is also infinitesimally jammed. Therefore the inner product between $x$ and $2 e_{i}$ does not change to first order, so $y_{i}=0$. Similarly $y_{j}=y_{k}=y_{\ell}=0$.

Now let $p \notin\{i, j, k, \ell\}$. By (2), there exists $b \in \mathcal{B}$ with $b_{p}=1$ and such that $b$ overlaps the support of $x$ in exactly two positions. Assume without loss of generality that $b$ is supported in positions $i, j, p$ and $q$. Then $\pm e_{i} \pm e_{j} \pm e_{p} \pm e_{q}$ is in $\mathcal{C}$ for all combinations of signs. The four points $x, z=-s_{i} e_{i}-s_{j} e_{j}-e_{p}-e_{q}, 2 e_{p}$ and $2 e_{q}$ span a scaled copy of the $D_{4}$ root system in $\mathcal{C}$; indeed, they form a $D_{4}$ Dynkin diagram:

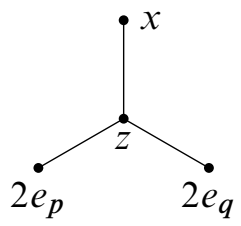

Therefore, the inner product between $x$ and $2 e_{p}$ does not change to first order, so $y_{p}=0$. It follows that $y=0$, and thus $\mathcal{C}$ is infinitesimally jammed.

The situation is quite different for lattice kissing configurations. The best one known in $\mathbb{R}^{10}$ is that of the lattice $\Lambda_{10}$; it contains 336 points and is not locally jammed (as one can check by a calculation like that for $\Lambda_{9}$ ). The best lattice kissing arrangement known in $\mathbb{R}^{11}$ comes from the lattice $\Lambda_{11}^{\max }$; it contains 438 points and is also not locally jammed.

The best lattice kissing configuration known in $\mathbb{R}^{12}$ is that of the Coxeter-Todd lattice $K_{12}$, which is not a laminated lattice. Unlike what happens in the previous three dimensions, this 756-point code is locally jammed and has a transitive symmetry group. However, we will show that it is not in fact jammed. Aside from the $A_{n}$ root systems, the Coxeter-Todd kissing configuration is the only example analyzed in this paper that is locally jammed but is not jammed. It is also remarkable because the Coxeter-Todd lattice is the densest sphere packing known in $\mathbb{R}^{12}$. This phenomenon of seemingly optimal packings with locally jammed yet unjammed kissing configurations also occurs in three dimensions, but we know of no other cases.

To unjam the Coxeter-Todd kissing configuration, we will make use of its Eisenstein structure: $K_{12}$ is a $\mathbb{Z}[\omega]$-module of dimension 6 , where $\omega$ is a primitive cube root of 
unity. We view $K_{12}$ as a subset of $\mathbb{C}^{6}$ with the inner product $\langle x, y\rangle=2 \operatorname{Re}\langle x, y\rangle_{\mathbb{C}}$, where $\langle z, w\rangle_{\mathbb{C}}=\sum_{j=1}^{6} z_{j} \bar{w}_{j}$ is the usual Hermitian inner product on $\mathbb{C}^{6}$. The minimal vectors of $K_{12}$ have norm 4 with respect to $\langle\cdot, \cdot\rangle$.

The key property of $K_{12}$ is that for minimal vectors $x$ and $y$ satisfying $x \neq \pm \omega^{j} y$, even the complex inner product $\langle x, y\rangle_{\mathbb{C}}$ is bounded in absolute value by 1 . This is quite unusual and does not hold for most other Eisenstein lattices (or related types of lattices such as Hurwitz lattices), but it can be checked directly from the list of minimal vectors in Conway and Sloane [19, page 128]. More conceptually, it follows from the Eisenstein integrality of $K_{12}$ (that is, the fact that all the complex inner products are in $\mathbb{Z}[\omega])$. Specifically, if $\left|\langle x, y\rangle_{\mathbb{C}}\right|>1$, then a quick enumeration shows that the only possibility is $\langle x, y\rangle_{\mathbb{C}}= \pm \omega^{j}(1-\omega)$, in which case $\left\langle x, \pm \omega^{j} y\right\rangle_{\mathbb{C}}=1-\omega$. However, a complex inner product of $1-\omega$ leads to a real inner product of 3 , which is impossible. Note that this property is an assertion about the minimal distance of the 126-point configuration in $\mathbb{C P} P^{5}$ obtained by taking the quotient of this code by the action of the multiplicative group $\mathbb{C}^{\times}$, which in this case just amounts to taking the quotient modulo the sixth roots of unity. In fact, it follows from Cohn and Kumar [10, Theorem 8.2] that the resulting code in $\mathbb{C} \mathbb{P}^{5}$ is universally optimal.

The Eisenstein structure breaks up the code into 126 hexagons (the orbits under multiplication by powers of the sixth root of unity $-\omega$ ), and to unjam it we simply rotate each of these hexagons by arbitrary angles. If we rotate $x$ to $e^{i \theta} x$ and $y$ to $e^{i \varphi} y$, then

$$
\left\langle e^{i \theta} x, e^{i \varphi} y\right\rangle_{\mathbb{C}}=e^{i(\theta-\varphi)}\langle x, y\rangle_{\mathbb{C}}
$$

and hence

$$
\left\langle e^{i \theta} x, e^{i \varphi} y\right\rangle=2 \operatorname{Re}\left\langle e^{i \theta} x, e^{i \varphi} y\right\rangle_{\mathbb{C}} \leq 2 \mid\left\langle e^{i \theta} x,\left.e^{i \varphi} y\right|_{\mathbb{C}}|=2|\langle x, y\rangle_{\mathbb{C}}\right| \leq 2 .
$$

If each hexagon is rotated by a slightly different angle, then this inequality will be strict whenever $x$ and $y$ lie in different hexagons. Therefore, we get an actual unjamming. Within each hexagon, the minimal distance has not changed. However, we can follow up such a deformation by moving the elements in each of the hexagons away from each other. For instance, decompose the hexagon into two equilateral triangles and move them away from each other, in a direction orthogonal to the plane of the hexagon. This process increases the minimal distance within each hexagon and results in a configuration of 756 points with minimal angle strictly larger than $\pi / 3$. Thus, we can not just unjam the $K_{12}$ kissing configuration, but unjam it so thoroughly that no contact remains (much like in three dimensions).

Note that it is difficult to predict results such as this based on dimension counting. For example, for large codes in $S^{n-1}$, the isostatic condition suggests that about $2(n-1)$ 
contacts per particle are needed to ensure jamming (see Torquato and Stillinger [44, page 2641]), because that yields $N(n-1)$ constraints for an $N$-particle code, which is the same as the number of degrees of freedom. (Strictly speaking, we should subtract $\operatorname{dim} O(n)=\left(\begin{array}{l}n \\ 2\end{array}\right)$ from the number of degrees of freedom, but that is negligible when $N$ is large.) For sufficiently generic constraints, one might expect this bound to be sharp, but it is far from sharp for highly symmetrical configurations. For example, each particle in the Coxeter-Todd kissing configuration is in contact with 82 others, so this configuration has far more contacts than the isostatic condition requires, but it is nevertheless unjammed. The $\Lambda_{9}$ kissing configuration also has plenty of contacts, but it is not even locally jammed.

\section{Kissing configuration of the Barnes-Wall lattice}

In this section, we show that the kissing configuration of the Barnes-Wall lattice is rigid. This lattice is the densest sphere packing known in $\mathbb{R}^{16}$, and it has the highest known kissing number.

We will consider the 4320 minimal vectors of $\Lambda_{16}$ as points on the sphere of radius 2 , which is their usual normalization. Then the inner products between distinct elements of the kissing configuration lie in the set $\{ \pm 2, \pm 1,0,-4\}$.

Recall that the minimal vectors of $\Lambda_{16}$ are as follows (see Conway and Sloane [19, page 129]). There are 480 of the form $2^{1 / 2}\left( \pm e_{i} \pm e_{j}\right)$ with $i \neq j$, which span $2^{1 / 2} D_{16}$, and 3840 of the form $2^{-1 / 2}\left(\sum_{i \in I} \pm e_{i}\right)$, where $I$ is the support of one of the 30 codewords of weight 8 in the first-order Reed-Muller code $\mathcal{R} \mathcal{M}(1,4)$ of block length 16 and where the number of minus signs is even. Codewords in $\mathcal{R M}(1,4)$ correspond to affine linear functions from $\mathbb{F}_{2}^{4}$ to $\mathbb{F}_{2}$, with the codeword consisting of the values of the function at the 16 points in $\mathbb{F}_{2}^{4}$.

Proposition 6.1 The kissing configuration of the Barnes-Wall lattice $\Lambda_{16}$ is infinitesimally jammed.

Proof Consider any infinitesimal deformation of this code. The points $2^{1 / 2}\left( \pm e_{i} \pm\right.$ $e_{j}$ ) form a copy of the $D_{16}$ root system (scaled by $2^{1 / 2}$ so the minimal distances match). This root system is infinitesimally jammed by Corollary 3.4, so after applying an infinitesimal rotation we can assume that all these points are fixed to first order. Furthermore, each pair of points with inner product \pm 2 spans a scaled copy of $A_{2}$, and each pair of antipodal points is contained in such a hexagon, so these inner products also do not change to first order. Thus, only the inner products 0 and \pm 1 can possibly change. 
We will now show that the facts in the previous paragraph together imply that the infinitesimal deformation must be identically zero. For consider a minimal vector $x=2^{-1 / 2} \sum_{i=1}^{8} s_{i} e_{k_{i}}$, where $1 \leq k_{1}<\cdots<k_{8} \leq 16$ and $s_{i} \in\{ \pm 1\}$, and suppose $x$ is infinitesimally perturbed by $y=\sum_{i} y_{i} e_{i}$.

The inner product of $x$ with the $D_{16}$ minimal vector $2^{1 / 2}\left(s_{i} e_{k_{i}}+s_{j} e_{k_{j}}\right)$ is 2 (note that the supports overlap and the signs cancel), and as noted above this inner product cannot change to first order. Because all the $D_{16}$ vectors are fixed, we find that $s_{i} y_{k_{i}}+s_{j} y_{k_{j}}=0$. This holds for every choice of $k_{i}$ and $k_{j}$, and if $k_{i}, k_{j}$ and $k_{\ell}$ are distinct, then the equations

$$
s_{i} y_{k_{i}}+s_{j} y_{k_{j}}=s_{i} y_{k_{i}}+s_{\ell} y_{k_{\ell}}=s_{j} y_{k_{j}}+s_{\ell} y_{k_{\ell}}=0
$$

imply that $y_{k_{i}}=y_{k_{j}}=y_{k_{\ell}}=0$. Since this holds for all $i=1, \ldots, 8$, we see that the nonzero coordinates of $x$ cannot change to first order. Next we have to show that the zero coordinates do not change either.

Let the positions of the zeros be $\ell_{1}<\cdots<\ell_{8}$, and consider the $D_{16}$ vector $z=$ $2^{1 / 2}\left(e_{\ell_{m}}+e_{\ell_{n}}\right)$ for some $m \neq n$. It is orthogonal to $x$, and by Lemma 6.2 below, $x$ and $z$ are contained in a sublattice isometric to a scaled copy of $D_{4}$. Since $D_{4}$ is infinitesimally jammed, the inner product $\langle x, z\rangle$ does not change to first order, and since $D_{16}$ is fixed, $z$ itself does not change to first order. Hence $y_{\ell_{m}}+y_{\ell_{n}}=0$. Similarly, $y_{\ell_{m}}-y_{\ell_{n}}=0$ by replacing $z$ with $z^{\prime}=2^{1 / 2}\left(e_{\ell_{m}}-e_{\ell_{n}}\right)$. Thus, $y_{\ell_{m}}=0$ for all $m$, and $x$ does not change to first order, as desired.

All that remains is to show that every pair of orthogonal minimal vectors in $\Lambda_{16}$ is contained in a scaled copy of $D_{4}$ (Lemma 6.2). This would be trivial if the automorphism group acted transitively on these pairs, because it is easy to exhibit a pair for which it is true (from a copy of $D_{4}$ inside $D_{16}$, for instance). However, there are actually two orbits. Each minimal vector in $\Lambda_{16}$ is orthogonal to 1710 others, and under the stabilizer of that vector they form two orbits, of size 1680 and 30 . This non-transitivity is actually quite remarkable, because it corresponds to a decomposition of the 4320 minimal vectors into 135 cross polytopes: to form one of the cross polytopes, take any vector, its antipode and the stabilizer orbit of size 30. It is far from obvious that this works, but it forms a decomposition that is invariant under the automorphism group. By contrast, the 240 minimal vectors in $E_{8}$ form 15 cross polytopes, and the 196560 in $\Lambda_{24}$ form 4095 (see, for example, Elkies [23, page 6, footnote 3]), but in these more symmetrical cases there is no invariant decomposition.

In principle, one could simply check Lemma 6.2 for the two orbits, but we will give a direct proof that requires less information about the action of the automorphism group. 
Lemma 6.2 Any two orthogonal vectors of $\Lambda_{16}$ are contained in a sublattice isometric to $D_{4}$ scaled by $2^{1 / 2}$.

Proof Let $x$ and $y$ be two orthogonal minimal vectors in $\Lambda_{16}$. Without loss of generality, we may assume that $x=2^{1 / 2}\left(e_{1}+e_{2}\right)$, because the automorphism group acts transitively on the minimal vectors (see Griess [25, Theorem 10.2] and [26]). We will construct vectors $w$ and $z$ such that $w, x, y, z$ form a $D_{4}$ Dynkin diagram, up to scaling:

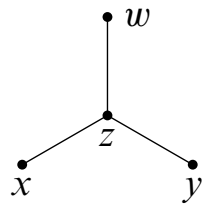

In other words, $\langle w, x\rangle=\langle x, y\rangle=\langle y, w\rangle=0$ and $\langle w, z\rangle=\langle x, z\rangle=\langle y, z\rangle=-2$. Then these vectors will span a copy of $2^{1 / 2} D_{4}$.

First, we analyze the case when $y \in 2^{1 / 2} D_{16}$. Then either $y= \pm 2^{1 / 2}\left(e_{1}-e_{2}\right)$ or $y=2^{1 / 2}\left( \pm e_{i} \pm e_{j}\right)$ with $2<i<j$. If $y= \pm 2^{1 / 2}\left(e_{1}-e_{2}\right)$, then we can assume the \pm sign is positive (by replacing $y$ with $-y$ if needed, since this does not change whether $x$ and $y$ are contained in a scaled copy of $\left.D_{4}\right)$, and we can take $z=2^{1 / 2}\left(e_{3}-e_{1}\right)$ and $w=2^{1 / 2}\left(e_{4}-e_{3}\right)$. If $y=2^{1 / 2}\left( \pm e_{i} \pm e_{j}\right)$ with $2<i<j$, then we can assume that $y=2^{1 / 2}\left(e_{i} \pm e_{j}\right)$ and take $z=-2^{1 / 2}\left(e_{1}+e_{i}\right)$ and $w=2^{1 / 2}\left(e_{1}-e_{2}\right)$.

In the remaining case, we have $y=2^{-1 / 2} \sum_{i=1}^{8} s_{i} e_{k_{i}}$ with $s_{i} \in\{ \pm 1\}$ and $k_{1}<k_{2}<$ $\cdots<k_{8}$. Since $x$ is orthogonal to $y$, either $k_{1}=1$ and $k_{2}=2$, or $k_{1}>2$. If $k_{1}=1$ and $k_{2}=2$, then $s_{1}+s_{2}=0$, and without loss of generality we can take $s_{1}=1$ and $s_{2}=-1$; then let $w=2^{1 / 2}\left(s_{3} e_{k_{3}}-s_{4} e_{k_{4}}\right)$ and $z=-2^{1 / 2}\left(e_{1}+s_{3} e_{k_{3}}\right)$.

Finally, suppose $k_{1}>2$. This case is slightly more subtle, because $z$ cannot be an element of $2^{1 / 2} D_{16}$, so we must instead produce a Reed-Muller codeword. The code $\mathcal{R M}(1,4)$ is five-dimensional, so we can find a nonzero codeword satisfying any four linear conditions. Specifically, we require it to vanish in coordinates $1,2, k_{1}$ and $k_{2}$. The resulting codeword $c$ has weight 8 , as do all nonconstant codewords.

The complementary codeword $(1, \ldots, 1)-c$ is also in $\mathcal{R} \mathcal{M}(1,4)$. Its support $\left\{\ell_{1}, \ldots, \ell_{8}\right\}$ contains $1,2, k_{1}$ and $k_{2}$, and it must intersect $\left\{k_{1}, \ldots, k_{8}\right\}$ in exactly four elements. Suppose $\ell_{1}=1, \ell_{2}=2$, and $\ell_{3}$ and $\ell_{4}$ are the other two elements of $\left\{\ell_{1}, \ldots, \ell_{8}\right\} \backslash$ $\left\{k_{1}, \ldots, k_{8}\right\}$. Now let $z=2^{-1 / 2} \sum_{i=1}^{8} t_{i} e_{\ell_{i}}$ with $t_{1}=t_{2}=-1$ and $t_{i}=-s_{j}$ whenever $\ell_{i}=k_{j}$. (The signs $t_{3}$ and $t_{4}$ can be chosen arbitrarily, subject to $z$ having a even number of minus signs.) Finally, let $w=-2^{1 / 2}\left(t_{3} e_{\ell_{3}}+t_{4} e_{\ell_{4}}\right)$. As in the previous cases, the vectors $w, x, y, z$ span a copy of $2^{1 / 2} D_{4}$, which completes the proof. 


\section{Kissing numbers in dimensions 25 through 31}

Before this paper, the best kissing numbers known in dimensions 25 through 31 were those shown in Table 3 (achieved by laminated lattices, see Conway and Sloane [15], with a correction from Musès [33]). These numbers increase surprisingly slowly from each dimension to the next, which made it difficult to believe they could be optimal, but they had not been improved since 1982, despite improvements starting in 32 dimensions from Edel, Rains and Sloane [22].

\begin{tabular}{cc} 
Dimension & Previous record \\
\hline 25 & 196656 \\
26 & 196848 \\
27 & 197142 \\
28 & 197736 \\
29 & 198506 \\
30 & 200046 \\
31 & 202692
\end{tabular}

Table 3: Previous record kissing numbers

All of these codes were based on the Leech lattice $\Lambda_{24}$ via a recursive construction in which each contains the previous one as a cross section. Recall that $\Lambda_{24}$ is the unique densest 24-dimensional lattice (see Cohn and Kumar [12]) and its kissing configuration of 196560 minimal vectors of norm 4 is the unique optimal 24-dimensional kissing arrangement (see Odlyzko and Sloane [36], Levenšteřn [29] and Bannai and Sloane [2]). The old 25-dimensional kissing number is just 96 more than the value achieved in $\mathbb{R}^{24}$, and it is obtained by putting cross polytopes above and below the Leech lattice kissing configuration. Specifically, in the coordinates used by Conway and Sloane [19, page 133], the Leech lattice contains a copy of $2^{1 / 2} D_{24}$, and to extend its kissing configuration to 25 dimensions one simply includes the remaining minimal vectors in $2^{1 / 2} D_{25}$. Much like the $\Lambda_{9}$ kissing configuration in $\mathbb{R}^{9}$ (which is constructed in a precisely analogous way from $E_{8}$ ), this code is not even locally jammed: all the points not in $2^{1 / 2} D_{25}$ can move. However, the analogy with 9 dimensions is not perfect, and the 25-dimensional version of Proposition 5.1 is not even true. We begin this section by showing how to improve the 25-dimensional kissing number while retaining the Leech lattice kissing configuration as a cross section. Once we have shown that, we will abandon the cross section constraint and go on to improve all the kissing numbers from 25 through 31 dimensions.

Given a vector $v \in \Lambda_{24}$ of norm 6 , consider the vectors $(2 v / 3, \pm 2 / \sqrt{3}) \in \mathbb{R}^{25}$, which lie on the same sphere (of radius 2 ) as the minimal vectors of the Leech lattice 
$\Lambda_{24} \times\{0\}$. If $x$ is any minimal vector in the Leech lattice, then $|v-x|^{2} \geq 4$, from which it follows that $\langle v, x\rangle \leq 3$. Thus,

$$
\langle(2 v / 3, \pm 2 / \sqrt{3}),(x, 0)\rangle \leq 2,
$$

so all the new vectors stay far enough away from those in the Leech lattice. However, they may come too close to each other if we use vectors $v$ that are too close. To avoid that problem, we choose a subset $R$ of the vectors of norm 6 in $\Lambda_{24}$ such that for $v, w \in R$ with $v \neq w$, we have $\langle v, w\rangle \leq 1$. Then

$$
\langle(2 v / 3, \pm 2 / \sqrt{3}),(2 w / 3, \pm 2 / \sqrt{3})\rangle \leq 4 / 9+4 / 3<2,
$$

as desired. Finally,

$$
\langle(2 v / 3,2 / \sqrt{3}),(2 v / 3,-2 / \sqrt{3})\rangle=4 \cdot 6 / 9-4 / 3<2 .
$$

Thus,

$$
\left\{(x, 0): x \in \Lambda_{24},|x|^{2}=4\right\} \cup\{(2 v / 3, \pm 2 / \sqrt{3}): v \in R\}
$$

achieves a kissing number of $196560+2|R|$ in $\mathbb{R}^{25}$.

We do not know how to maximize the size of $R$, but greedy approaches easily yield $|R|>48$ via a computer search and thus improve the kissing number. The best we have achieved is $|R|=70$, and the vectors are listed in the supplementary information (see Appendix A). If we take into account that all the inner products are in $\{-6,-4,-3,-2, \pm 1,0\}$, then linear programming bounds (see Delsarte, Goethals and Seidel [20]) prove that $|R| \leq 280$. Thus, our construction of $R$ cannot be improved by more than a factor of four, but we do not know how close to optimal it is.

We do not have a conceptual explanation of the 70-point configuration produced by the computer search, but we do have a simple construction that achieves $|R|=56$, which is enough to improve the kissing number in $\mathbb{R}^{25}$.

Proposition 7.1 There is an antipodal set $R$ of vectors of norm 6 in $\Lambda_{24}$ such that $|R|=56$ and $|\langle x, y\rangle| \leq 1$ for all distinct, non-antipodal $x, y \in R$.

Proof The Leech lattice $\Lambda_{24}$ contains two orthogonal copies of the Coxeter-Todd lattice $K_{12}$ (see Conway and Sloane [19, page 128, equations (129) and (130)]). Thus, it suffices to find such a subset of size 28 within $K_{12}$. To do so, we will use the construction of $K_{12}$ given in Section 2.5 of Conway and Sloane [16, Section 2.5, page 426], which is called $\Lambda^{(7)}$ in that paper.

This construction realizes $K_{12}$ as an Eisenstein lattice, that is, a $\mathbb{Z}[\omega]$-module with $\omega=e^{2 \pi i / 3}$. Let $\alpha=2+3 \omega$, so $|\alpha|^{2}=7$. Then the Coxeter-Todd lattice $K_{12}$ is 
isometric to $L$, where

$$
L=\frac{1}{\alpha}\left\{\left(x_{1}, \ldots, x_{7}\right) \in \mathbb{Z}[\omega]^{7}: x_{1} \equiv x_{2} \equiv \cdots \equiv x_{7}(\bmod \alpha) \text { and } \sum_{j=1}^{7} x_{j}=0\right\} .
$$

As before, we use the inner product $\langle x, y\rangle=2 \operatorname{Re}\langle x, y\rangle_{\mathbb{C}}$, where $\langle z, w\rangle_{\mathbb{C}}=\sum_{j=1}^{7} z_{j} \bar{w}_{j}$ is the usual Hermitian inner product on $\mathbb{C}^{7}$.

We will make use of only some of the vectors of norm 6 in $L$, namely those obtained by permuting the coordinates of $\left(1, \omega, \omega^{2}, 0,0,0,0\right)$. Let $W$ be the set of such points. Note that $1+\omega+\omega^{2}=0$ and $\alpha \equiv \alpha \omega \equiv \alpha \omega^{2} \equiv 0(\bmod \alpha)$, so these vectors are indeed in $L$.

We begin by finding a six-dimensional regular simplex in $K_{12}$ (that is, seven vectors of norm 6 whose inner products are all -1 ). To do so, start by choosing seven vectors in $W$ such that the supports of any two overlap in exactly one coordinate position. Equivalently, label the seven positions by points in the Fano plane $\mathbb{P}^{2}\left(\mathbb{F}_{2}\right)$, and choose the seven lines in that plane as the supports of the vectors.

Suppose two of these vectors overlap in position $i$, with coordinates $\omega^{j}$ and $\omega^{k}$. Then their inner product in $L$ is $2 \operatorname{Re} \omega^{j-k}$, which equals -1 if and only if $j \neq k$. Thus, we must ensure that each pair of vectors differ in the position where they overlap.

Equivalently, labeling the incident point-line pairs in $\mathbb{P}^{2}\left(\mathbb{F}_{2}\right)$ with $1, \omega$ or $\omega^{2}$ must yield a three-coloring of the edges of the bipartite incidence graph between the points and lines (the Heawood graph). The existence of such a coloring follows from Konnig's line coloring theorem (see Lovász and Plummer [30, Theorem 1.4.18]), which says that the chromatic index of a bipartite graph equals its maximal degree. It is not difficult to exhibit such a coloring, and hence a six-dimensional simplex in $K_{12}$, explicitly as the rows of the following array:

$\begin{array}{ccccccc}0 & 1 & 0 & \omega & 0 & \omega^{2} & 0 \\ 1 & 0 & 0 & \omega^{2} & \omega & 0 & 0 \\ 0 & 0 & \omega & 1 & 0 & 0 & \omega^{2} \\ \omega & \omega^{2} & 1 & 0 & 0 & 0 & 0 \\ 0 & \omega & 0 & 0 & \omega^{2} & 0 & 1 \\ \omega^{2} & 0 & 0 & 0 & 0 & 1 & \omega \\ 0 & 0 & \omega^{2} & 0 & 1 & \omega & 0\end{array}$

To produce 14 of the desired 28 points in $L$, we simply take these seven points and their antipodes. The remaining 14 can be obtained from them by permuting the coordinates. 
Specifically, we fix the seventh coordinate and permute the others via the six-cycle

$$
1 \mapsto 6 \mapsto 4 \mapsto 3 \mapsto 2 \mapsto 5 \mapsto 1
$$

or its inverse. It is not difficult to check that if we apply either permutation to the original simplex in $L$, then the seven new points all have inner products 0 or \pm 1 with the original points.

Thus, we have found 28 points in $K_{12}$ with the desired inner products, and hence 56 in $\Lambda_{24}$.

In the remainder of this section, we will further improve the records for kissing numbers in dimensions 25 through 31 . All of our constructions will be quite simple, except that they rely on finding a subset $S$ of the minimal vectors in $\Lambda_{24}$ such that no two elements of $S$ have inner product greater than 1. We will give a conceptual argument that achieves $|S|=288$, which will be enough to improve all the kissing numbers. Further optimization using a computer has led to an antipodal subset with $|S|=480$, which yields the new records shown in Table 1. The code of size 480 is enumerated in the accompanying computer files (see Appendix A). If we take into account that all the inner products are in $\{-4,-2, \pm 1,0\}$, then linear programming bounds prove that $|S| \leq\lfloor 9360 / 11\rfloor=850$.

Lemma 7.2 There is an antipodal set $S$ of minimal vectors in $\Lambda_{24}$ such that $|S|=288$ and $|\langle x, y\rangle| \leq 1$ for all distinct, non-antipodal $x, y \in S$.

The code we construct below is relatively well known, but for completeness we sketch the construction here.

Proof Let $\mathcal{N R}$ be the Nordstrom-Robinson code, a nonlinear code of size 256 and distance 6 in $\mathbb{F}_{2}^{16}$ (see MacWilliams and Sloane [31, page 73]). Let $W$ consist of all the vectors $\left(\sum_{i}(-1)^{s_{i}} e_{i}\right) / 2$ with $\left(s_{1}, \ldots, s_{16}\right) \in \mathcal{N} \mathcal{R}$, as well as the vectors $\pm 2 e_{i}$ for $1 \leq i \leq 16$. The elements of $W$ have norm 4 , and is easy to check that distinct vectors of $W$ have inner product at most 1 with each other. One can also check that the span of $W$ is isomorphic to the Barnes-Wall lattice. Since there is an embedding of the Barnes-Wall lattice into the Leech lattice (see Conway and Sloane [15]) we obtain the desired subset $S$ of $\Lambda_{24}$ as the image of $W$.

For the rest of this section, $S$ will denote any set of minimal vectors in $\Lambda_{24}$ such that $\langle x, y\rangle \leq 1$ for all distinct $x, y \in S$. In particular, it may be the set from Lemma 7.2, the one of size 480 found by a computer search, or an even larger set. Also, let $\mathcal{C}$ denote the set of minimal vectors in $\Lambda_{24}$. 
Proposition 7.3 The kissing number in dimension 25 is at least $196560+|S|$.

Proof Fix an angle $\theta$ (to be determined shortly), and let

$$
T=\{(x, 0): x \in \mathcal{C} \backslash S\} \cup\{(x \cos \theta, \pm 2 \sin \theta): x \in S\} .
$$

In other words, we remove $S$ from the equatorial hyperplane and replace it with two copies lying on the parallels at latitude $\pm \theta$.

The inner product between a vector $(x, 0)$ and $(y \cos \theta, \pm 2 \sin \theta)$ is $\langle x, y\rangle \cos \theta \leq 2$, since $x \neq y$ (and hence $\langle x, y\rangle \leq 2$ ).

The inner product between two distinct vectors in the northern hemisphere is

$$
\begin{aligned}
\langle(x \cos \theta, 2 \sin \theta),(y \cos \theta, 2 \sin \theta)\rangle & =\langle x, y\rangle \cos ^{2} \theta+4 \sin ^{2} \theta \\
& \leq \cos ^{2} \theta+4 \sin ^{2} \theta \\
& =1+3 \sin ^{2} \theta,
\end{aligned}
$$

which is at most 2 provided that $\sin ^{2} \theta \leq 1 / 3$.

The inner product between two vectors in opposite hemispheres is

$$
\begin{aligned}
\langle(x \cos \theta, 2 \sin \theta),(y \cos \theta,-2 \sin \theta)\rangle & =\langle x, y\rangle \cos ^{2} \theta-4 \sin ^{2} \theta \\
& \leq 4 \cos ^{2} \theta-4 \sin ^{2} \theta \\
& \leq 4-8 \sin ^{2} \theta
\end{aligned}
$$

which is at most 2 provided that $\sin ^{2} \theta \geq 1 / 4$. Therefore, for any choice of $\theta$ such that $1 / 4 \leq \sin ^{2} \theta \leq 1 / 3$, the code $T$ of size $196560+|S|$ has maximal inner product 2 and is thus a kissing configuration.

To deal with higher dimensions, we will use disjoint sets $S_{1}, \ldots, S_{51} \subset \mathcal{C}$ with the same property that $\langle x, y\rangle \leq 1$ for all distinct $x, y \in S_{i}$.

Lemma 7.4 Let $S_{1}=S$. Then there are disjoint subsets $S_{2}, S_{3}, \ldots \subset \mathcal{C}$ such that $\langle x, y\rangle \leq 1$ for all distinct $x, y \in S_{i}$ and

$$
\left|S_{i}\right| \geq|S|\left(1-\frac{\sum_{j=1}^{i-1}\left|S_{j}\right|}{196560}\right) .
$$

Furthermore, if $S$ is antipodal, then we can take $S_{2}, S_{3}, \ldots$ to be antipodal as well. 
Of course, $S_{i}=\varnothing$ if $i$ is sufficiently large. The proof of Lemma 7.4 uses the probabilistic method, rather than an explicit construction. This approach is surely not optimal, but it is convenient. Furthermore, it naturally leads to a probabilistic algorithm that can be used to obtain these sets with the aid of a computer.

Proof Let $G$ be the automorphism group of the Leech lattice. We will obtain the sets $S_{2}, S_{3}, \ldots$ by induction as subsets of translates of $S$ by elements of $G$. First, we compute the expected size of the intersection of $g S$ with $S_{1} \cup \cdots \cup S_{i-1}$ when $g$ is chosen uniformly at random from $G$. Since $S_{1}, \ldots, S_{i-1}$ are disjoint, we have

$$
\underset{g \in G}{\mathbb{E}}\left|g S \cap\left(S_{1} \cup S_{2} \cdots \cup S_{i-1}\right)\right|=\sum_{j=1}^{i-1} \mathbb{E}_{g \in G}\left|g S \cap S_{j}\right| \text {. }
$$

The terms on the right side are easily computed, via

$$
\begin{aligned}
\mathbb{E}_{g \in G}\left|g S \cap S_{j}\right| & =\sum_{x \in S} \operatorname{Pr}_{g \in G}\left[g x \in S_{j}\right] \\
& =\sum_{x \in S} \sum_{y \in S_{j}} \operatorname{Pr}_{g \in G}[g x=y] \\
& =\frac{|S|\left|S_{j}\right|}{196560} .
\end{aligned}
$$

In the last line, we have used the fact that $G$ acts transitively on the 196560 minimal vectors; therefore, $g x$ is uniformly distributed among those vectors as $g$ varies, and

$$
\underset{g \in G}{\operatorname{Pr}}[g x=y]=\frac{1}{196560} \text {. }
$$

Now there must be a translate $g S$ whose overlap with $S_{1} \cup \cdots \cup S_{i-1}$ is at most the expectation, namely

$$
|S| \frac{\sum_{j=1}^{i-1}\left|S_{j}\right|}{196560} \text {. }
$$

Letting $S_{i}=g S \backslash \bigcup_{j=1}^{i-1} S_{j}$ completes the proof. (Note that if $S$ is antipodal, then each of these sets will be as well.)

Theorem 7.5 Let $S_{1}, S_{2}, \ldots$ be as in Lemma 7.4. Suppose we have partitioned a kissing configuration in the unit sphere $S^{d-1} \subset \mathbb{R}^{d}$ into subsets $T_{1}, \ldots, T_{N}$ such that for distinct $x, y \in T_{i}$, we have $\langle x, y\rangle \leq-1 / 2$. Then the kissing number in dimension $24+d$ is at least

$$
\sum_{i=1}^{N}\left(\left|T_{i}\right|-1\right)\left|S_{i}\right|
$$


and it is achieved by the set

$$
\left\{(x, 0) \in \mathbb{R}^{24} \times \mathbb{R}^{d}: x \in \mathcal{C} \backslash \bigcup_{i=1}^{N} S_{i}\right\} \cup \bigcup_{i=1}^{N}\left\{(x \sqrt{2 / 3}, y \sqrt{4 / 3}): x \in S_{i}, y \in T_{i}\right\} .
$$

Note that the minimal angle constraint on $T_{i}$ implies that $\left|T_{i}\right| \leq 3$, and $\left|T_{i}\right|=3$ is possible only if $T_{i}$ consists of three points forming an equilateral triangle on a great circle. Of course it is pointless to take $\left|T_{i}\right|=1$, and to optimize the bound we should take $\left|T_{1}\right| \geq\left|T_{2}\right| \geq \cdots \geq\left|T_{N}\right|$, assuming $\left|S_{1}\right| \geq\left|S_{2}\right| \geq \cdots \geq\left|S_{N}\right|$.

Proof First, observe that the points $(x \sqrt{2 / 3}, y \sqrt{4 / 3})$ have norm

$$
\frac{2}{3}|x|^{2}+\frac{4}{3}|y|^{2}=\frac{2}{3} \cdot 4+\frac{4}{3} \cdot 1=4,
$$

as they should. Within each set $\left\{(x \sqrt{2 / 3}, y \sqrt{4 / 3}): x \in S_{i}, y \in T_{i}\right\}$, the inner product between two distinct points $(x \sqrt{2 / 3}, y \sqrt{4 / 3})$ and $\left(x^{\prime} \sqrt{2 / 3}, y^{\prime} \sqrt{4 / 3}\right)$ is at most

$$
\frac{2}{3} \cdot 4-\frac{4}{3} \cdot \frac{1}{2}=2
$$

if $y \neq y^{\prime}$, and at most

$$
\frac{2}{3} \cdot 1+\frac{4}{3} \cdot 1=2
$$

if $x \neq x^{\prime}$. Inner products between two of these sets are also easily dealt with: for $i \neq j$, the inner product between $(x \sqrt{2 / 3}, y \sqrt{4 / 3})$ with $x \in S_{i}, y \in T_{i}$ and $\left(x^{\prime} \sqrt{2 / 3}, y^{\prime} \sqrt{4 / 3}\right)$ with $x^{\prime} \in S_{j}, y^{\prime} \in T_{j}$ is at most

$$
\frac{2}{3} \cdot 2+\frac{4}{3} \cdot \frac{1}{2}=2 \text {. }
$$

Finally, the inner product between $(x \sqrt{2 / 3}, y \sqrt{4 / 3})$ and $\left(x^{\prime}, 0\right)$ is at most $2 \sqrt{2 / 3}$, which is strictly less than 2 .

Note that the code from Theorem 7.5 cannot be even locally jammed, provided that $\mathcal{C} \neq \bigcup_{i=1}^{N} S_{i}$, because the points in

$$
\left\{(x, 0) \in \mathbb{R}^{24} \times \mathbb{R}^{d}: x \in \mathcal{C} \backslash \bigcup_{i=1}^{N} S_{i}\right\}
$$

are not adjacent to any of the points of the code that are not in this set.

We do not know the best way to apply Theorem 7.5, but we can use it as follows. Given any kissing configuration in $\mathbb{R}^{d}$ of size $K$, we want to partition it into antipodal pairs and equilateral triangles (or singletons if necessary). Of course, if it is antipodal we can simply partition it into $K / 2$ antipodal pairs, but that will generally not be optimal. 


\begin{tabular}{cccc} 
Dimension & Lower bound & $|S|=288$ & $|S|=480$ \\
\hline 25 & $196560+\left|S_{1}\right|$ & 196848 & 197040 \\
26 & $196560+2\left|S_{1}\right|+2\left|S_{2}\right|$ & 197712 & 198480 \\
27 & $196560+2\left|S_{1}\right|+2\left|S_{2}\right|+\sum_{i=3}^{5}\left|S_{i}\right|$ & 198576 & 199912 \\
28 & $196560+2 \sum_{i=1}^{8}\left|S_{i}\right|$ & 201156 & 204188 \\
29 & $196560+2 \sum_{i=1}^{8}\left|S_{i}\right|+\sum_{i=9}^{16}\left|S_{i}\right|$ & 203430 & 207930 \\
30 & $196560+2 \sum_{i=1}^{24}\left|S_{i}\right|$ & 210200 & 219008 \\
31 & $196560+2 \sum_{i=1}^{24}\left|S_{i}\right|+\sum_{i=25}^{51}\left|S_{i}\right|$ & 217588 & 230872
\end{tabular}

Table 4: New lower bounds on kissing numbers

If there is an Eisenstein structure, as is the case for $A_{2}, D_{4}, E_{6}$ and $E_{8}$, then we partition it into regular hexagons using that structure and divide each hexagon into two equilateral triangles. For $A_{3}, D_{5}$ and $E_{7}$, we partition a cross section using its Eisenstein structure and then fill the rest with antipodal pairs. This yields the bounds shown in Table 4. To compute $\left|S_{i}\right|$, we simply use Lemma 7.4 recursively, taking into account that $\left|S_{i}\right|$ must be not just an integer, but also even when $S_{i}$ is antipodal. For example, if $|S|=480$ with $S$ antipodal, then $\left|S_{2}\right| \geq 480 \cdot(1-480 / 196560) \approx 478.83$, which implies $\left|S_{2}\right|=480$.

We do not expect that these bounds are anywhere close to being optimal, and already in $\mathbb{R}^{32}$ they are worse than the previous record: they give 266544, while the record is 276032 ( $|S|=554$ would be required to break the record). However, our bounds improve upon all cases from dimension 25 to 31 .

\section{Open problems}

We conclude with some open problems. The most basic is whether there are spherical codes that span the ambient space and are jammed but not infinitesimally jammed. We suspect that there are such codes, but we have not found one. A related question is how to test efficiently whether a code is jammed (which would be possible if jamming and infinitesimal jamming were equivalent, although one could hope for even faster algorithms). Such an algorithm might be based on higher-order variants of infinitesimal jamming, but they are far more subtle than one might expect (see Connelly and Servatius [14]).

Our new kissing records in dimensions 25 through 31 can presumably be improved, and it would be very interesting to know how far these techniques can be pushed, or how 
to construct much better arrangements. The ratio of the upper bounds from Shtrom [40] to the lower bounds proved here grows roughly like $1.4^{d}$ in $24+d$ dimensions for $1 \leq d \leq 7$, so there is considerable room for improvement. Note that optimizing the sizes of the sets $R$ and $S$ from Section 7 can be viewed as maximizing the size of cliques in highly symmetrical graphs. Finding large cliques is NP-hard in general, but that does not settle the question of how well one can solve this problem in practice.

Among kissing problems in low dimensions, dimensions 17 through 23 seem ripe for improvement, although it is unlikely that the approach we have used here can be made to work. Dimension 16 is particularly interesting, and we would very much like to know whether the 4320 minimal vectors of the Barnes-Wall lattice solve the kissing problem. They are certainly not the unique solution, because we have found that at least one of the 16-dimensional packings constructed by Conway and Sloane [18] has a different kissing configuration of the same size. It seems unlikely that one could classify all the possibilities in $\mathbb{R}^{16}$, but it might be possible to extend the conjecturally exhaustive list from Section 4 to higher dimensions, perhaps up through $\mathbb{R}^{12}$.

In Section 5 we gave a list of the best kissing configurations known in $\mathbb{R}^{9}$ through $\mathbb{R}^{12}$, but we suspect even more such configurations remain to be found. As mentioned in that section, the $E_{7}$ root system fits into the same framework. In fact its three other competitors do too, in the following sense. In each case, one can find a cross polytope contained in the configuration (for example, by a randomized, greedy algorithm). Using it to define the coordinate system yields simple rational coordinates for each point. In the $E_{7}$ case, these coordinates come from a constant weight code. In the other three cases they do not, but the coordinates can be obtained by a systematic mutation of the $E_{7}$ case. Perhaps one could obtain additional kissing configurations in nine through twelve dimensions via a similar construction.

The problem of rigidity naturally generalizes to many other ambient spaces, such as projective spaces, but this generalization presents new and interesting features. For example, a projective configuration is not determined by its pairwise distance matrix, and in complex projective space there are even continuous families of optimal codes with exactly the same pairwise distances (see Cohn and Kumar [10, page 129]).

Even in Euclidean space, which is the most thoroughly studied case so far, there are unresolved questions. How can one test whether a periodic packing is jammed? If one restricts attention to packings consisting of $N$ translates of a lattice (that is, those with $N$ particles per unit cell), then one can test for infinitesimal jamming and hence jamming (see Donev, Torquato, Stillinger and Connelly [21]). However, the answer may depend on $N$. For example, the laminated lattice $\Lambda_{9}$ is jammed with $N=1$ (that is, it cannot be deformed as a lattice packing), but not with $N=2$ (giving rise to the 
fluid diamond packings of Conway and Sloane [18]). We know of no bound for how large $N$ must be to detect a lack of rigidity.

Finally, we conjecture that in all sufficiently high dimensions, there exist optimal kissing configurations with no contacts whatsoever (that is, no pairs of points with inner product $1 / 2$ ), so they are unjammed in the strongest possible sense. This phenomenon occurs in $\mathbb{R}^{3}$, but we know of no higher-dimensional cases. Part of our motivation for making this conjecture is that we know of no large, jammed kissing configurations at all in high dimensions. The $D_{n}$ root system is jammed for $n>3$, but it contains only $2 n(n-1)$ points, which is tiny compared with the exponential growth of the kissing number (see, for example, Conway and Sloane [19, pages 23-24]). Are there exponentially large jammed kissing configurations in high dimensions? Are there even any of greater than quadratic size? For example, is the kissing configuration of the Barnes-Wall lattice $B W_{2^{k}}$ in $\mathbb{R}^{2^{k}}$ always jammed? (It has size asymptotic to $C \cdot 2^{k(k+1) / 2}$, where $C$ is a constant [19, page 24].)

\section{Acknowledgements}

We thank Károly Bezdek, Noam Elkies, Michel Goemans and Achill Schürmann for helpful discussions, Patric Östergård for providing computer files for Best's constant weight codes, and an anonymous referee for providing useful feedback on the manuscript. Abhinav Kumar was supported in part by National Science Foundation grants DMS-0757765 and DMS-0952486 and by a grant from the Solomon Buchsbaum Research Fund, and he thanks Princeton University for its hospitality. Yang Jiao and Salvatore Torquato were supported in part by NSF grants DMS-0804431 and DMR-0820341.

\section{Appendix A Data files}

As supplementary information for this paper, we have made available sixteen data files through the arXiv.org e-print archive, where it is paper number arXiv:1102.5060. One can access these files by downloading the source files for the paper. The data files can also be downloaded from the web page for this article (doi:10.2140/gt.2011.15.2235).

Ten of them describe the kissing configurations enumerated in Section 4. These files are each named after the corresponding configuration: 5-40a.txt, 5-40b.txt, 6-72a.txt through 6-72d.txt, and 7-126a.txt through 7-126d.txt. The first line of the file specifies the number $N$ of points, and the second line specifies the dimension $n$ of the ambient Euclidean space. The third line consists of $n$ positive 
integers $d_{1}, \ldots, d_{n}$ (separated by spaces), which are the coefficients of the diagonal quadratic form used to measure distances. Finally, the remaining $N$ lines each give the $n$ coordinates of one of the points in the configuration (again separated by spaces). The scaling has been chosen so that all the coordinates will be integers, and the inner product between points $x$ and $y$ is defined by

$$
\langle x, y\rangle=\sum_{i=1}^{n} d_{i} x_{i} y_{i} .
$$

Equivalently, if we use the standard inner product, then the $i$ th coordinate must be scaled by $\sqrt{d_{i}}$, but phrasing it in terms of changing the inner product avoids the need to use irrational numbers as coordinates. (There exist different coordinate systems that use only rational numbers, even with the standard inner product, but the coordinates used here are compatible with the fibering construction from Section 4.) Note that within each file, all the vectors have the same norm, but they are not unit vectors.

Four of the files, namely b9-18.txt, b10-30.txt, b11-35.txt and b12-51.txt, describe the constant weight binary codes used to build the kissing configurations from Table 2 in Section 5. The file bn-N.txt contains all the codes of block length $n$, size $N$, constant weight 4 and minimal distance 4 , up to isomorphism. (We thank Patric Östergård for providing these codes.) Each codeword is given on a line by itself, with the binary digits separated by spaces, and there is a blank line between different codes.

The remaining two files, R.txt and S.txt, describe the 70-point configuration $R$ and the 480-point configuration $S$ from Section 7. These files are in a slightly different format: they omit the first three lines $\left(N, n\right.$ and $\left.d_{1}, \ldots, d_{n}\right)$. Instead, each line specifies the 24 coordinates of one of the points in the coordinate system used by Conway and Sloane [19, page 133, Figure 4.12], but with the irrational factor of $1 / \sqrt{8}$ omitted.

\section{References}

[1] D L Applegate, W Cook, S Dash, D G Espinoza, Exact solutions to linear programming problems, Oper. Res. Lett. 35 (2007) 693-699 MR2361036

[2] E Bannai, N J A Sloane, Uniqueness of certain spherical codes, Canad. J. Math. 33 (1981) 437-449 MR617634

[3] J A Barrau, On the combinatory problem of Steiner, Proc. Section of Sciences, Koninklijke Akademie van Wetenschappen te Amsterdam 11 (1908) 352-360

[4] M R Best, $A[11,4,4]=35$ or some new optimal constant weight codes, Technical Report ZN 71/77, Mathematisch Centrum, Amsterdam (1977) Available at http:// repository.cwi.nl/search/fullrecord.php?publnr $=7433$ 
[5] MR Best, Binary codes with minimum distance four, Technical Report ZW 112/78, Mathematisch Centrum, Amsterdam (1978) Available at http:// repository.cwi.nl/search/fullrecord.php?publnr $=6831$

[6] M R Best, Binary codes with a minimum distance of four, IEEE Trans. Inform. Theory 26 (1980) 738-742 MR596287

[7] A Bezdek, K Bezdek, R Connelly, Finite and uniform stability of sphere packings, Discrete Comput. Geom. 20 (1998) 111-130 MR1626699

[8] K Böröczky, Jr, Finite packing and covering, Cambridge Tracts in Mathematics 154, Cambridge University Press, Cambridge (2004) MR2078625

[9] H Cohn, Order and disorder in energy minimization, from: "Proceedings of the International Congress of Mathematicians, Hyderabad, August 19-27, 2010, Volume IV", Hindustan Book Agency, New Delhi (2010) 2416-2443

[10] H Cohn, A Kumar, Universally optimal distribution of points on spheres, J. Amer. Math. Soc. 20 (2007) 99-148 MR2257398

[11] H Cohn, A Kumar, Counterintuitive ground states in soft-core models, Phys. Rev. E (3) 78 (2008) 061113:1-7 MR2546058

[12] H Cohn, A Kumar, Optimality and uniqueness of the Leech lattice among lattices, Ann. of Math. (2) 170 (2009) 1003-1050 MR2600869

[13] R Connelly, The rigidity of certain cabled frameworks and the second-order rigidity of arbitrarily triangulated convex surfaces, Adv. Math. 37 (1980) 272-299 MR591730

[14] R Connelly, H Servatius, Higher-order rigidity — what is the proper definition?, Discrete Comput. Geom. 11 (1994) 193-200 MR1254089

[15] J H Conway, N J A Sloane, Laminated lattices, Ann. of Math. (2) 116 (1982) 593-620 MR678483

[16] J H Conway, N J A Sloane, The Coxeter-Todd lattice, the Mitchell group, and related sphere packings, Math. Proc. Cambridge Philos. Soc. 93 (1983) 421-440 MR698347

[17] J H Conway, N J A Sloane, The cell structures of certain lattices, from: "Miscellanea mathematica", Springer, Berlin (1991) 71-107 MR1131118

[18] J H Conway, N J A Sloane, What are all the best sphere packings in low dimensions?, Discrete Comput. Geom. 13 (1995) 383-403 MR1318784

[19] J H Conway, NJ A Sloane, Sphere packings, lattices and groups, third edition, Grundlehren der Mathematischen Wissenschaften 290, Springer, New York (1999) MR1662447

[20] P Delsarte, J M Goethals, J J Seidel, Spherical codes and designs, Geom. Dedicata 6 (1977) 363-388 MR0485471

[21] A Donev, S Torquato, F H Stillinger, R Connelly, A linear programming algorithm to test for jamming in hard-sphere packings, J. Comput. Phys. 197 (2004) 139-166 MR2061244 
[22] Y Edel, EM Rains, N J A Sloane, On kissing numbers in dimensions 32 to 128, Electron. J. Combin. 5 (1998) R22 MR1614304

[23] ND Elkies, Mordell-Weil lattices in characteristic 2 II: The Leech lattice as a MordellWeil lattice, Invent. Math. 128 (1997) 1-8 MR1437492

[24] V Elser, S Gravel, Laminating lattices with symmetrical glue, Discrete Comput. Geom. 43 (2010) 363-374 MR2579702

[25] R L Griess, Jr, Pieces of $2^{d}$ : existence and uniqueness for Barnes-Wall and Ypsilanti lattices, Adv. Math. 196 (2005) 147-192 MR2159298

[26] R L Griess, Jr, Corrections and additions to: "Pieces of $2^{d}$ : existence and uniqueness for Barnes-Wall and Ypsilanti lattices” [Adv. Math. 196 (2005) 147-192], Adv. Math. 211 (2007) 819-824 MR2323546

[27] J Leech, Five dimensional non-lattice sphere packings, Canad. Math. Bull. 10 (1967) 387-393 MR0220170

[28] J Leech, Six and seven dimensional non-lattice sphere packings, Canad. Math. Bull. 12 (1969) 151-155 MR0254746

[29] V I Levenšteĭn, On bounds for packings in n-dimensional Euclidean space, Dokl. Akad. Nauk SSSR 245 (1979) 1299-1303 MR529659 (Russian) English translation in Soviet Math. Dokl. 20 (1979) 417-421

[30] L Lovász, M D Plummer, Matching theory, AMS Chelsea Publishing, Providence, RI (2009) MR2536865 Corrected reprint of the 1986 original

[31] F J MacWilliams, N J A Sloane, The Theory of Error-correcting Codes, North-Holland Mathematical Library 16, North-Holland Publishing Co., Amsterdam-New YorkOxford (1977) MR465509

[32] H D Mittelmann, F Vallentin, High-accuracy semidefinite programming bounds for kissing numbers, Experiment. Math. 19 (2010) 175-179 MR2676746

[33] C Musès, The dimensional family approach in (hyper)sphere packing: a typological study of new patterns, structures, and interdimensional functions, Appl. Math. Comput. 88 (1997) 1-26 MR1473570

[34] O R Musin, The kissing number in four dimensions, Ann. of Math. (2) 168 (2008) 1-32 MR2415397

[35] G Nebe, NJA Sloane, Table of the highest kissing numbers presently known Available at http://www.math.rwth-aachen.de/ Gabriele.Nebe/LATTICES/ kiss.html

[36] A M Odlyzko, NJ A Sloane, New bounds on the number of unit spheres that can touch a unit sphere in $n$ dimensions, J. Combin. Theory Ser. A 26 (1979) 210-214 MR530296

[37] P R J Östergård, Classification of binary constant weight codes, IEEE Trans. Inform. Theory 56 (2010) 3779-3785 MR2752466 
[38] B Roth, W Whiteley, Tensegrity frameworks, Trans. Amer. Math. Soc. 265 (1981) 419-446 MR610958

[39] K Schütte, B L van der Waerden, Das Problem der dreizehn Kugeln, Math. Ann. 125 (1953) 325-334 MR0053537

[40] D V Shtrom, The Delsarte method in the problem of the contact numbers of Euclidean spaces of high dimensions, Proc. Steklov Inst. Math. (2002) supplement 2, S162-S189 MR2068195

[41] T Tarnai, Zs Gáspár, Improved packing of equal circles on a sphere and rigidity of its graph, Math. Proc. Cambridge Philos. Soc. 93 (1983) 191-218 MR691989

[42] A Tarski, A decision method for elementary algebra and geometry, second edition, University of California Press, Berkeley and Los Angeles, CA (1951) MR0044472

[43] S Torquato, F H Stillinger, Multiplicity of generating, selection, and classification procedures for jammed hard-particle packings, J. Phys. Chem. B 105 (2001) 1184911853

[44] S Torquato, F H Stillinger, Jammed hard-particle packings: from Kepler to Bernal and beyond, Rev. Modern Phys. 82 (2010) 2633-2672

[45] G L Watson, The number of minimum points of a positive quadratic form, Dissertationes Math. (Rozprawy Mat.) 84 (1971) MR0318061

[46] V A Zinoviev, T Ericson, New lower bounds for contact numbers in small dimensions, Problemy Peredachi Informatsii 35 (1999) 3-11 MR1737742 (Russian) English translation in Problems Inform. Transmission 35 (1999) 287-294

Microsoft Research New England

One Memorial Drive, Cambridge, MA 02142

Physical Sciences-Oncology Center, Princeton University

Princeton, New Jersey 08544

Department of Mathematics, Massachusetts Institute of Technology

Cambridge, MA 02139

Department of Chemistry, Princeton University

Princeton, New Jersey 08544

cohn@microsoft.com, yjiao@princeton.edu, abhinav@math.mit.edu, torquato@electron.princeton.edu

Proposed: Rob Kirby

Seconded: Dmitri Burago, Joan Birman
Received: 24 February 2011

Revised: 23 May 2011 